\title{
Belphégor
}

\section{Du journal illustré au magazine moderne : l'hebdomadaire VU (1928-1940), un effort de moyennisation de l'avant-garde?}

\section{Laura Truxa}

\author{
(2) OpenEdition \\ Journals \\ Édition électronique \\ URL : https://journals.openedition.org/belphegor/4194 \\ DOI : 10.4000/belphegor.4194 \\ ISSN : 1499-7185 \\ Éditeur \\ LPCM
}

Référence électronique

Laura Truxa, « Du journal illustré au magazine moderne : I'hebdomadaire VU (1928-1940), un effort de moyennisation de l'avant-garde? », Belphégor [En ligne], 19-2 | 2021, mis en ligne le 05 janvier 2022, consulté le 20 septembre 2022. URL : http://journals.openedition.org/belphegor/4194 ; DOI : https:// doi.org/10.4000/belphegor.4194

Ce document a été généré automatiquement le 20 septembre 2022.

\section{(c) (i) (9)}

Creative Commons - Attribution - Pas d'Utilisation Commerciale - Pas de Modification 4.0 International - CC BY-NC-ND 4.0

https://creativecommons.org/licenses/by-nc-nd/4.0/ 


\title{
Du journal illustré au magazine moderne : l'hebdomadaire $V U$ (1928-1940), un effort de moyennisation de l'avant-garde?
}

\author{
Laura Truxa
}

L'hebdomadaire d'information VU (1928-1940) fut longtemps connu comme un exemple parmi d'autres de ces illustrés pionniers du photojournalisme, tels que Regards ou Voilà. Ce n'est que récemment qu'il a fait l'objet d'études approfondies, par le biais principal de l'histoire de la photographie ${ }^{1}$. Le rôle des photographes qui ont contribué à son iconographie, et dont certainees ont intégré le canon de l'histoire de l'art, a été souligné. L'étude ici présentée se propose quant à elle, dans un premier temps, d'insister sur la proximité de $V U$ avec un réseau d'artistes et typographes d'avantgarde, impliqués dans le développement de nouvelles formes de communication graphique. Le périodique partage ainsi des similarités avec les œuvres de l'Union des Artistes Modernes, du Bauhaus et de la Nouvelle Typographie. Or, le réseau des producteurs de VU réunit également, de part et d'autre de l'Atlantique, des entreprises innovantes aux intérêts étroitement liés, et appartenant au secteur des communications de masse. Pour mieux appréhender $V U$ et ses spécificités sur le marché de la presse, il convient d'examiner ses liens avec la fonderie Deberny \& Peignot, l'agence de publicité Dorland et les publications de Condé Nast. On s'appuiera principalement sur les travaux récents consacrés à $V U$, ainsi que sur un ensemble de sources diverses comprenant des publications professionnelles, des annuaires et catalogues industriels, et les biographies et autobiographies des collaborateur.ice's de $V U$. Par ses qualités matérielles, mais également par sa ligne éditoriale, VU est le produit des exigences à la fois esthétiques et marchandes de ses créateur.ice-s. Son design peut être envisagé, enfin, comme un instrument de séduction de nouvelles catégories de consommateurice's de presse au sein des classes moyennes françaises. De ce point de vue, l'hebdomadaire élabore une formule médiatique originale, définie en grande partie par son style visuel dérivé du modernisme artistique. Ni revue 
bourgeoise, ni illustré populaire, $V U$ signale l'avènement du format magazine, véhicule par excellence d'une culture industrielle « moyenne » ou middlebrow.

\section{Un projet médiatique nourri de l'influence des avant- gardes}

2 Lorsqu'il crée $V U$, Lucien Vogel est un éditeur confirmé qui bénéficie d'une vingtaine d'années d'expérience ${ }^{2}$. L'hebdomadaire est stratégiquement présenté comme la réponse à un manque au sein du paysage médiatique français, énoncé dès l'éditorial de son premier numéro, "Remarques sur un nouveau journal illustré $»^{3}$ : situé entre les quotidiens, les journaux d'opinion, les « somptueux magazines » de mode ou d'art, les illustrés sportifs et les publications littéraires et humoristiques, $V U$ se donne pour mission de synthétiser les multiples facettes de « la vie contemporaine ». Il s'intéresse à l'actualité politique, culturelle, sportive ou vestimentaire, au tourisme ou encore à la vulgarisation scientifique, et accorde une place importante aux feuilletons. Cependant, l'aspect le plus remarquable du projet est sa dimension visuelle. Amateur d'art et figure importante de la vie culturelle parisienne, Vogel imagine un journal « animé comme un beau film $»^{4}$, à la pointe de l'innovation esthétique et technique. Pour servir cet objectif, il mobilise un réseau international de créateurice's proches de l'avant-garde artistique, et s'inspire de la presse illustrée allemande et suisse ${ }^{5}$, imprimée en héliogravure. Ce procédé, introduit avant la guerre en France par L'Illustration ${ }^{6}$, permet une grande souplesse de mise en page et un rendu très fin des images photographiques, qui deviennent la marque de fabrique de $V U$.

Fig.1. VU, 1, 21 mars 1928, pp. 6-7. Format : $54 \times 37 \mathrm{~cm}$. Reproduction : Musée Nicéphore Niépce, Ville de Chalon-sur-Saône.

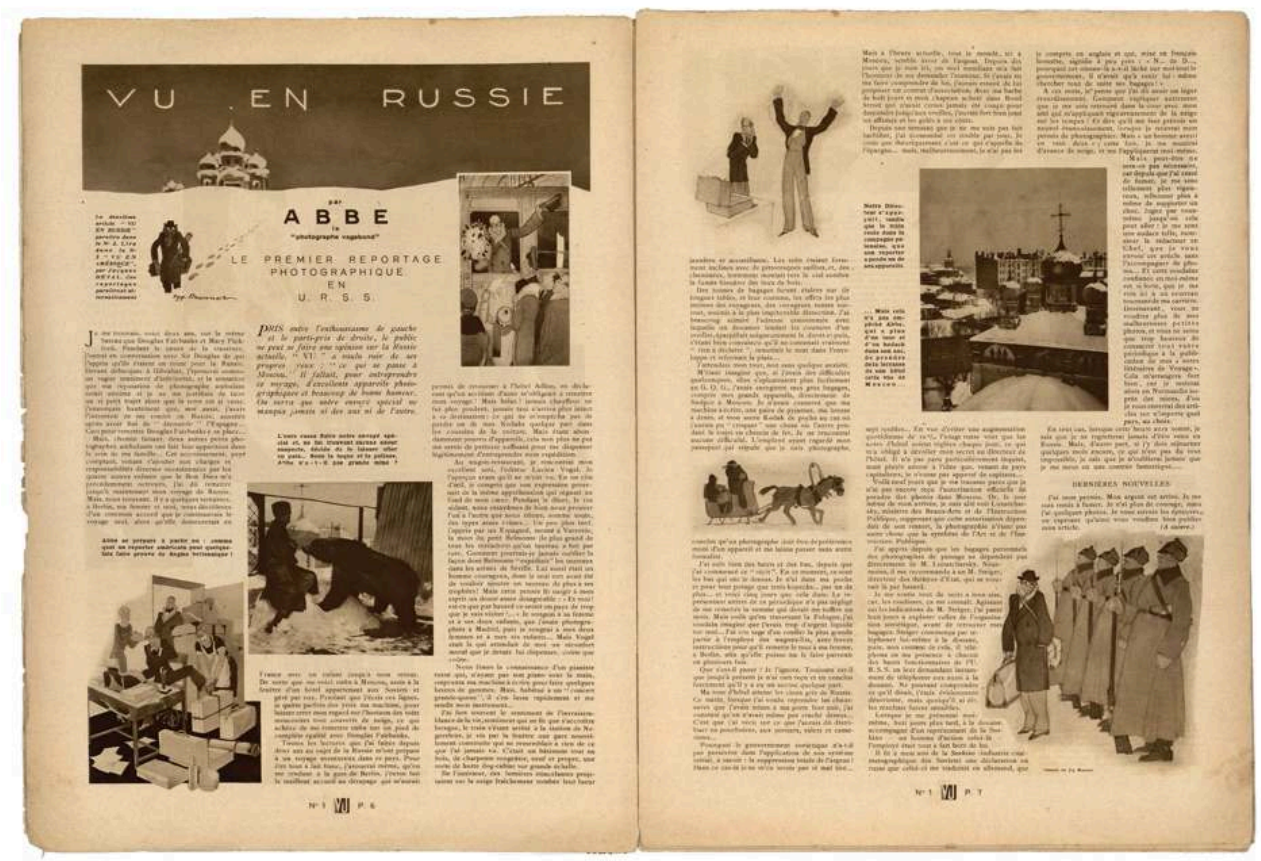

3 Vogel soigne ainsi l'apparence de sa publication et commande des images aux artistes qu'il fréquente, tels que Germaine Krüll, Man Ray ou André Kertész. La mise en page 
évolue au fil du temps, et rend compte d'un apprentissage progressif de son mode de composition par ses concepteurice-s. Ancienne élève et collaboratrice de l'affichiste Alexey Brodovitch, Irène Lidova est embauchée à l'atelier de $V U$ pour coloriser les photographies de couverture. Elle y apprend la mise en page auprès de Vogel, et rapidement y obtient la « première place " ${ }^{7}$. À ses débuts, $V U$ ressemble encore, comme l'annonce son sous-titre, à un journal. Sa composition suit une grille classique, dont les colonnes de texte symétriques laissent place çà et là aux rectangles parfaitement perpendiculaires d'illustrations que bordent de larges marges blanches (Fig. 1). Quelques années plus tard, la diversité et l'asymétrie ont dynamité la grille du VU des premiers temps. L'unité de composition devient la double-page, au travers de laquelle se déploient les images et la titraille (Fig. 2). Les marges sont réduites, et disparaissent souvent tout à fait, submergées par des photographies imprimées «à bords perdus » (Fig. 3). Les titres monumentaux imposent des effets d'échelle et des jeux typographiques qui tiennent davantage de l'affiche que de l'édition. Les lettres sans empattement viennent concurrencer le digne elzévir du corps de texte. Cette évolution est peut-être due en partie à l'arrivée d'Alexander Liberman, recruté en 1933 par Vogel alors qu'il est assistant d'A. M. Cassandre, auteur du logotype de VU. Toutefois, bien qu'il revendique le statut de directeur artistique ${ }^{8}$, Liberman est formé par Lidova, qu'il est à l'origine chargé de seconder'.

Fig.2. VU, 368, 3 avril 1935, pp. 430-431. Format : $54 \times 37 \mathrm{~cm}$. Reproduction : Musée Nicéphore Niépce, Ville de Chalon-sur-Saône.

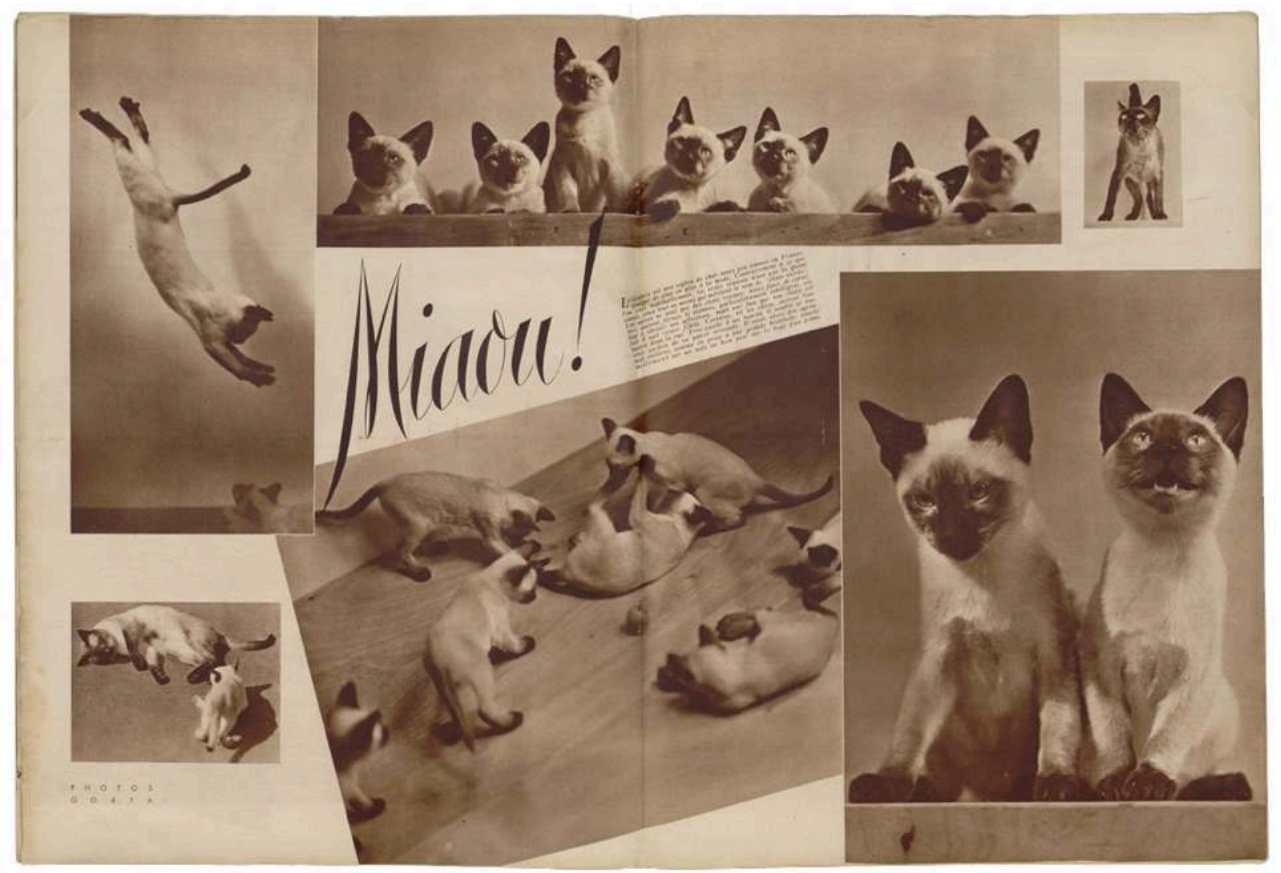


Fig.3. VU, Hors-Série «Salon de l'automobile », $1^{\text {er }}$ octobre 1933, pp. 36-37. Format : $54 \times 37 \mathrm{~cm}$. Reproduction : Musée Nicéphore Niépce, Ville de Chalon-sur-Saône.

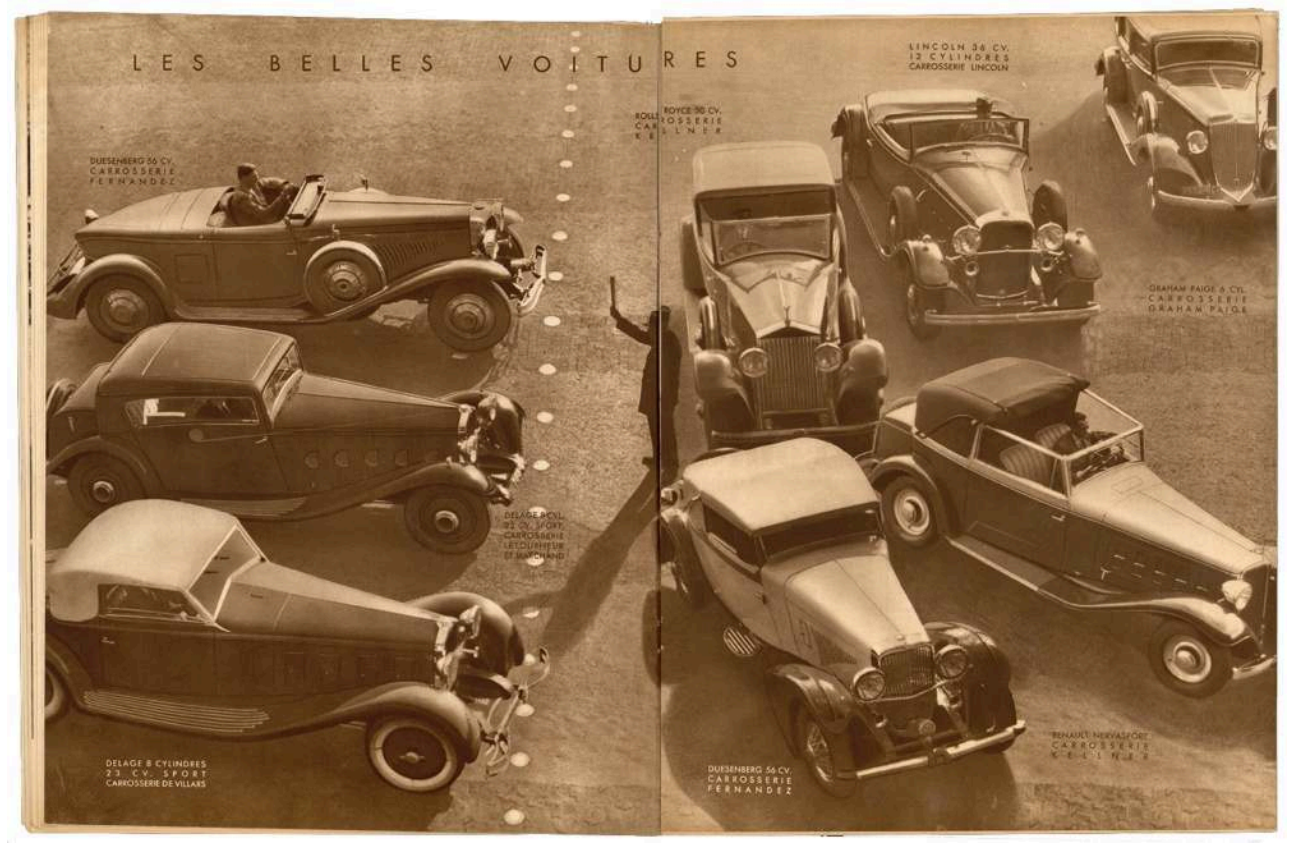

La grande diversité des caractères typographiques qui composent $V U$ est le fruit d'un accès privilégié à l'abondant catalogue de la fonderie Deberny \& Peignot (D\&P), première sur le marché typographique français ${ }^{10}$. En effet, une comparaison du périodique et des Spécimens Généraux édités en 1926 et en 1935 révèle que tous les caractères employés par $V U$, à l'exception de ceux de ses annonces publicitaires, en sont tirés. Charles Peignot, héritier et directeur artistique de D\&P, crée avec Vogel en 1927 la revue professionnelle Arts et métiers graphiques (AMG). Publicitaires, imprimeurs, photographes y défendent des conceptions nouvelles de leurs métiers. Peignot y soutient la protection légale des caractères, qui ferait du typographe un artiste doté de son style propre ${ }^{11}$. Brodovitch contribue à introduire le terme "graphisme" pour désigner l'art des affichistes ${ }^{12}$. Nombreux sont les collaborateurs d'AMG, tels que Jean Carlu, Cassandre, Peignot lui-même ou Maximilien Vox, le chef du service typographique de D\&P, à rejoindre l'Union des Artistes Modernes (UAM) ${ }^{13}$. Celle-ci milite pour un art utile et industriel, débarrassé des hiérarchies entre arts majeurs et mineurs et de l'esthétique ornementale qui triomphe à l'Exposition des Arts Décoratifs de 1925. La revue est également ouverte à la création internationale. En 1930, Jan Tschichold y présente les principes de la Nouvelle Typographie, mouvement lié au Bauhaus dont il est le principal théoricien ${ }^{14}$.

5 La Nouvelle Typographie réunit des typographes exerçant en Allemagne, pour la plupart issus des arts plastiques. Selon eux, la communication graphique doit s'adapter avec rationalité aux conditions de vie du monde moderne urbanisé, en suivant les principes de "réduction formelle » et le rejet de l'ornement hérités de la peinture abstraite et du constructivisme. Ils privilégient les contrastes visuels, les compositions asymétriques, et le recours, dans la mesure du possible, à une seule casse : un texte devrait idéalement être composé entièrement en lettres majuscules ou, mieux encore, entièrement en minuscules ${ }^{15}$. Comme le préconise László Moholy-Nagy, qui est le premier à employer l'expression "Nouvelle Typographie» dans le catalogue de l'exposition du Bauhaus de $1923^{16}$, les Nouveaux Typographes ont volontiers recours à 
l'illustration photographique, au photomontage et à la «typophoto $»^{17}$. Ils apprécient les formes géométriques d'allure industrielle et les caractères typographiques sans empattements. Le Futura, dessiné par Paul Renner et édité en 1927 par la fonderie Bauer est un des plus grands succès de ce courant. En 1930, D\&P en obtient les droits français, après avoir refusé un projet de caractère original proposé par Tschichold ${ }^{18}$. Le Futura intègre dès juillet la maquette de VU (Fig. 4) (Fig. 5), qui adopte peu à peu, au cours des années suivantes, nombre des principes de composition chers à la Nouvelle Typographie.

Fig.4. Le caractère typographique Futura, dessiné par Paul Renner et édité en France par la fonderie Deberny \& Peignot, en usage sur la couverture de VU, 407, 2 janvier 1936 . Format : $27 \times 37 \mathrm{~cm}$.

Reproduction : Musée Nicéphore Niépce, Ville de Chalon-sur-Saône.

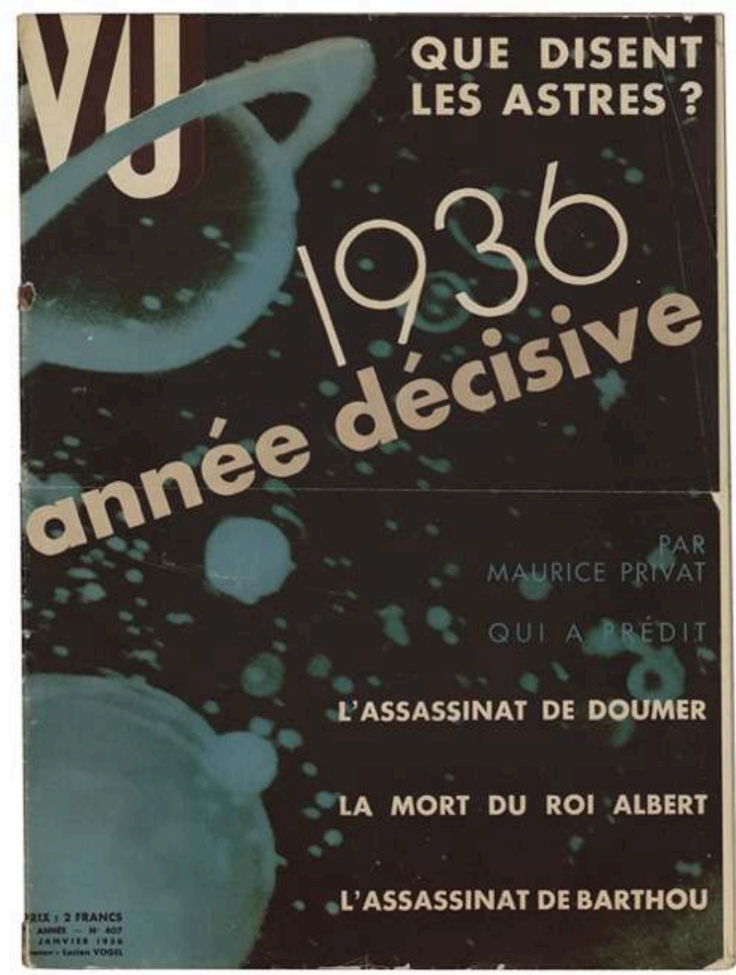


Fig.5. Le caractère typographique Futura, dessiné par Paul Renner et édité en France par la fonderie Deberny \& Peignot, en usage sur une double-page de VU, Hors-Série " 29 e Salon de l'auto ", 30 septembre 1935, pp. 30-31. Format : $54 \times 37 \mathrm{~cm}$. Reproduction : Musée Nicéphore Niépce, Ville de Chalon-sur-Saône.

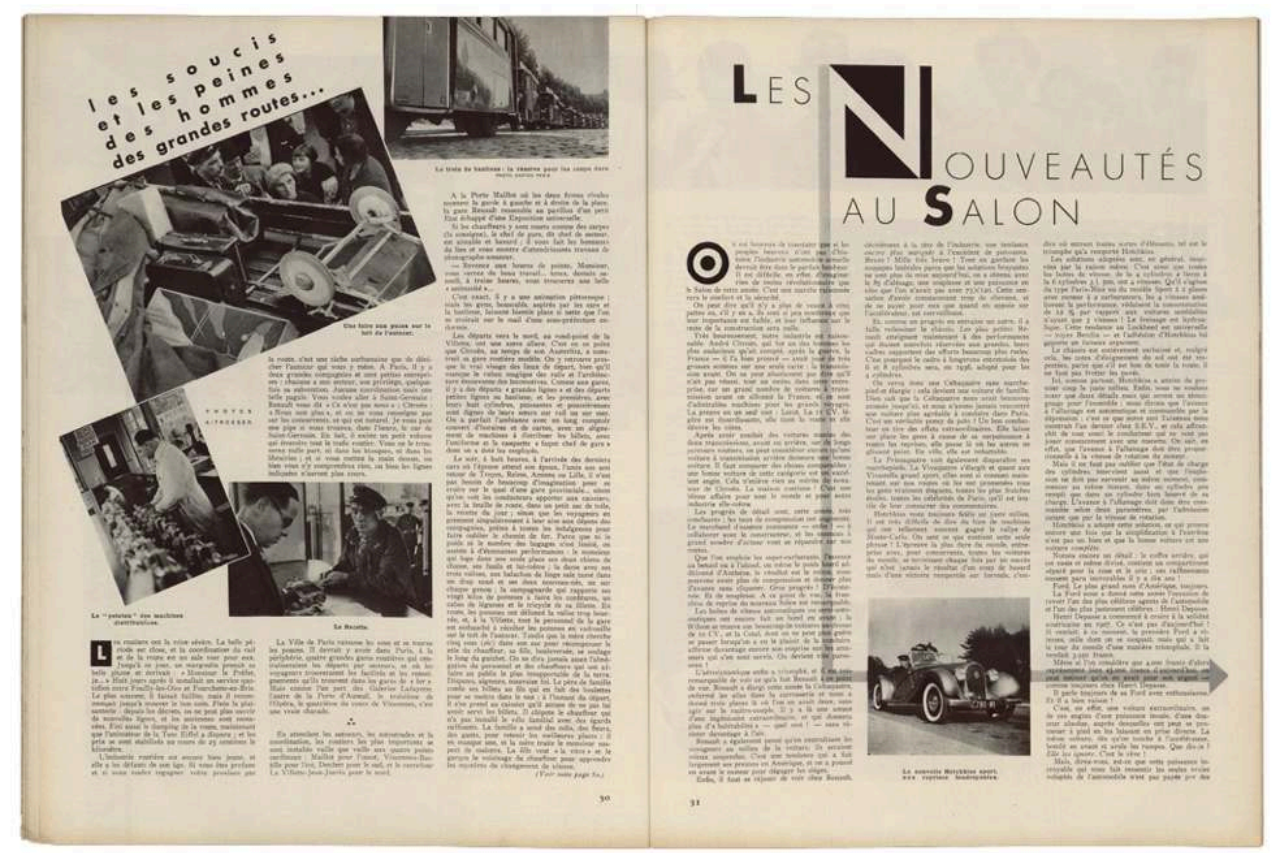

6 Ces échanges témoignent de la reconnaissance mutuelle qui lie les avant-gardes européennes et les cercles auxquels appartiennent les contributeurice's de VU. Le périodique est d'ailleurs présenté en 1928 à l'Exposition Internationale de la Presse de Cologne, dite Pressa ${ }^{19}$. Outre l'exposition constructiviste du pavillon soviétique, réalisée par El Lissitzky, Pressa accueille plusieurs Nouveaux Typographes dans le pavillon de la presse ouvrière ${ }^{20}$ et dans l'exposition du Bauhaus «Elementare Buchteknik $»^{21}$. Vogel et son équipe ont ainsi de toute évidence pleine connaissance des innovations esthétiques qui ont cours outre-Rhin dans le domaine de la mise en page. Lors du départ de Vogel en 1936 suivi de la démission de Liberman en 1937, VU est nettement plus "moderne ", visuellement, qu'il ne l'était en 1928. Il l'est également davantage que la plupart des autres hebdomadaires d'information français, de L'Illustration et du Miroir du monde à Regards ou Voilà. Si ces derniers se montrent inventifs, ils ne manifestent pas la même prodigalité typographique. Cependant, certaines compositions de $V U$, et en particulier celles des numéros hors-séries, font preuve d'une audace graphique ou d'un sens du pastiche qui ne sont guère représentatifs de la maquette habituelle de l'hebdomadaire (Fig. 6).

7 De même, certaines photographies peuvent s'avérer décevantes pour quiconque y chercherait le style singulier de leurs auteur.ice's aujourd'hui célèbres. $V U$ ne devrait pas en ce sens être assimilé à un périodique d'avant-garde. 
Fig.6. Deux double-pages imitant le style constructiviste dans un numéro spécialement consacré à I'URSS. VU, 192, 18 novembre 1931, pp. $2544-2545 ; 2576-2577$. Format : $54 \times 37 \mathrm{~cm}$.

Reproduction : Musée Nicéphore Niépce, Ville de Chalon-sur-Saône.
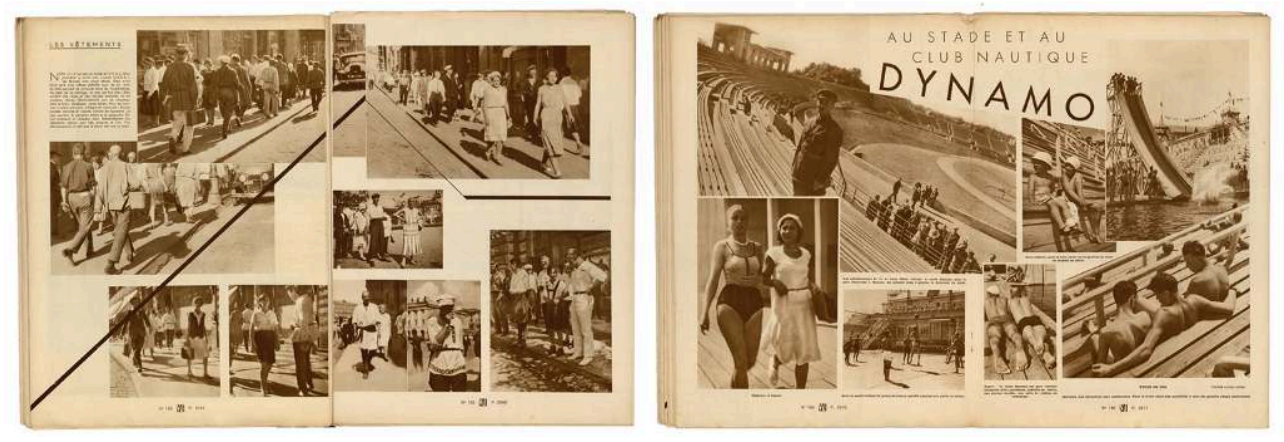

\section{Un réseau d'entreprises aux pratiques commerciales et publicitaires innovantes}

En outre, les circonstances de la publication de VU suggèrent que son appropriation de procédés graphiques dits modernistes découle d'une volonté stratégique de s'associer à un style en vogue. En effet, l'hebdomadaire s'inscrit dans un réseau d'entreprises liées aux secteurs de l'édition et de la publicité qui font de l'exploitation de la «tendance » dérivée du Bauhaus leur fonds de commerce.

Le premier nœud de ce réseau est le duo formé par Vogel et Peignot. Peignot est actionnaire de la Société des Illustrés Français qui édite $V U$ et siège à son conseil d'administration ${ }^{22}$. De son côté Vogel est co-fondateur et actionnaire de la société Arts et métiers graphiques ${ }^{23}$; il siège à son conseil d'administration et fait partie du comité de direction de la revue, dirigée par Peignot. Chacun profite donc, littéralement, du succès de l'autre, et a tout intérêt à y veiller. $A M G$ contient presque à chaque numéro plusieurs annonces et articles qui vantent les mérites des productions de D\&P et de son atelier de photographie publicitaire, le Studio D\&P, ouvert en 1929. Aussi la revue fonctionne-t-elle comme une vitrine de l'entreprise de Peignot. VU peut également être envisagé comme un vecteur de diffusion des produits D\&P, ce qui expliquerait la qualité et la diversité inhabituelles des caractères typographiques qui le composent : il permet de faire connaître les nouvelles tendances graphiques à un public bien plus large que celui d'une publication professionnelle ${ }^{24}$. Les lecteur.ice's de l'hebdomadaire sont des consommateur-ice's dont l'attention est convoitée par les éditeurs et les publicitaires, eux-mêmes lecteurs d'AMG et donc, clients potentiels de D\&P. En somme, cet échange de bons procédés permettrait à l'hebdomadaire d'être toujours "à la page " et à la fonderie de publiciser massivement ses produits. L'intérêt de Peignot pour la Nouvelle Typographie n'est pas davantage désintéressé. Suite à l'échec commercial du Bifur de Cassandre, la fonderie D\&P a besoin en 1929 d'un nouveau caractère de labeur pour renflouer ses caisses ${ }^{25}$. L'achat des droits du Futura, qui connaît un grand succès en Allemagne, est donc au moins autant lié à des considérations économiques qu'esthétiques.

10 Vogel forme également une alliance avec Condé Nast, pour qui il représente une porte d'entrée sur le marché de la presse européenne. Dès 1915, Nast cherche à s'impliquer financièrement dans la luxueuse Gazette du Bon Ton, première création de Vogel ${ }^{26}$. Il confie en 1920 le lancement de l'édition française de Vogue à Vogel, qui en est le 
directeur artistique de 1925 à 1927, et à son épouse Cosette Vogel de Brunhoff, qui en est la rédactrice en chef jusqu'en 1927. Puis, en 1922, il rachète le supplément de L'Illustration édité par Vogel depuis 1920 sous le titre L'Illustration des Modes, rebaptisé Le Jardin des $\operatorname{Modes}^{27}$. En parallèle, Nast fait entrer sur la scène parisienne Walter Seymour Maas, cadre de l'agence de publicité Dorland. Fondée en 1883 à Atlantic City, Dorland ouvre un bureau londonien en 1905, qui est chargé de la régie publicitaire européenne de Condé Nast Publications et de la distribution de Vogue sur le territoire britannique. En 1916, Nast décide de créer une première édition internationale de Vogue au Royaume-Uni. Ce sont les experts de Dorland, dont Maas, qui sont alors responsables non seulement de sa régie, mais également de sa gestion complète ${ }^{28}$. Lorsqu'est créée l'édition française de Vogue, Maas ouvre pour Dorland un bureau parisien dont la principale activité en est la régie publicitaire ${ }^{29}$. Celles du Jardin des Modes puis de VU lui sont ensuite confiées lors de la création de ces titres ${ }^{30}$. En 1928, l'agence qui se présente comme «la plus puissante organisation mondiale de publicité " $^{31}$ s'installe en même temps que VU et Vogue au 65-67, avenue des Champs-Élysées. Ainsi, les trois entreprises partagent la même adresse et sont liées au point que les frontières, à la fois physiques et professionnelles, en sont floues, ce dont témoignera ultérieurement Liberman ${ }^{32}$. Maas fait également partie du comité de direction de la revue $A M G$, ce qui suggère une possible collaboration entre l'agence Dorland, dont les activités incluent la conception graphique d'annonces, et le studio ou la fonderie D\&P, fournisseur de matériel typographique. Certaines annonces de Dorland en France sont d'ailleurs composées en Futura ${ }^{33}$.

11 À partir des années 1930, Condé Nast Publications et Dorland mobilisent leurs relations européennes pour grossir les rangs de leurs maisons-mères, à l'instar d'autres entreprises étasuniennes qui profitent des vagues d'émigration de la décennie pour se renouveler et affûter leurs techniques de vente ${ }^{34}$. En 1928 est créée une édition allemande de Vogue avec la complicité de l'antenne berlinoise de Dorland. Le directeur artistique de celle-ci, Mehemed Fehmy Agha, est chargé de la conception visuelle de la revue. Après l'échec commercial du Vogue allemand, Agha émigre en 1929 à New-York et est nommé directeur artistique des publications américaines de Condé Nast ${ }^{35}$. À la suite de son passage au Vogue allemand ou peu avant, Dorland recrute le Nouveau Typographe Herbert Bayer, qui quitte alors la direction du département de l'imprimé et de la publicité du Bauhaus de Dessau ${ }^{36}$. L'agence joue pendant quelques années un rôle important pour l'insertion professionnelle des anciens étudiants et enseignants de l'école ${ }^{37}$. C'est dans ce contexte, en 1929 , que lui est confiée la régie publicitaire du mensuel die neue linie, considéré aujourd'hui comme un exemple paradigmatique de «domestication» du graphisme de la Nouvelle Typographie ${ }^{38}$. Sa maquette originelle, imaginée par Moholy-Nagy et Bayer, est probablement réalisée en partie par les assistants de ce dernier à Dorland ${ }^{39}$. En 1938, Bayer rejoint l'agence new-yorkaise de Dorland, puis fait carrière sur Madison Avenue, épicentre de l'empire publicitaire américain ${ }^{40}$. Il contribue notamment à l'image de marque de la Container Corporation of America, aux côtés de Cassandre, Carlu, Man Ray et Moholy-Nagy, qui participe également à la création de l'école de design de Chicago sous le nom initial de New Bauhaus $^{41}$. En 1941, Liberman s'installe lui aussi aux États-Unis. Sur la recommandation de Lidova, il rencontre Brodovitch devenu en 1934 le directeur artistique du magazine Harper's Bazaar. Sur celle de Vogel, qui est alors consultant auprès de Condé Nast, il se fait recruter au sein du département d'Agha ${ }^{42}$. Ce dernier est peu après remercié, et en 1943 Liberman hérite du poste de directeur artistique de $V_{o g u} e^{43}$. Le réseau 
professionnel de $V U$ atteste donc d'une grande porosité des frontières entre les mondes de l'art et ceux de l'industrie et des médias, mais également d'une circulation ininterrompue de leurs acteurs, à la fois nationale et internationale, intrinsèquement liée au développement d'une culture de la consommation de masse ${ }^{44}$.

Le projet d'adapter l'inventivité de l'avant-garde au profit du commerce, qui sous-tend les activités de Nast, Dorland, Vogel et D\&P, est en effet caractéristique de la période. L'historien de l'art Frederic Schwartz a par exemple démontré la manière dont les objets du Bauhaus sont, presque dès ses débuts, saisis par le marché et imités, donnant lieu à un "style Bauhaus " dérivatif. À l'occasion des dix ans de l'institution, les commentateurs raillent déjà les dames de la bonne société berlinoise qui distribuent lors de leurs cocktail parties des cartes de visite composées intégralement en «bas de casse $»^{45}$. Julia Meer a quant à elle conclu, à partir d'une étude de la presse professionnelle allemande des milieux de l'imprimerie et de la publicité, que plusieurs aspects des créations des Nouveaux Typographes, dont les compositions diagonales et les formes géométriques, sont repris par les publicitaires, en tant que mode décorative ou ornementale ${ }^{46}$. Les thèses utopistes de la Nouvelle Typographie sur la rationalisation de la lecture, en revanche, n'intéressent guère les professionnels. Le marché français s'empare lui aussi des créations des artistes modernes. Ceux-ci s'associent à des spécialistes du commerce, souhaitant rationaliser et moderniser leurs pratiques de réclame, pour défendre l'affiche, présentée comme une nouvelle forme artistique ${ }^{47}$. L'UAM, dont VU est proche, est particulièrement impliquée dans cette alliance. Lors de l'Exposition Internationale de 1937, nombreux sont ses membres à exposer leurs travaux dans le pavillon dédié à la publicité, conçu par René Herbst et Carlu. Elle contribue également au Salon des Arts Ménagers ${ }^{48}$, créé en 1923, qui fait de l'espace domestique un enjeu créatif. Dans ce contexte culturel transnational inédit, VU est un pôle parisien qui met en lien des hommes d'affaires, des artistes, des graphistes, des publicitaires, et des photographes. Il prend ainsi part à l'élaboration des professions et des savoir-faire nouveaux bientôt associés à la publicité et au design, inscrivant le souci esthétique au cœur de la production industrielle.

\section{Une formule éditoriale et graphique " moyenne »}

13 Eu égard à cette dynamique de commercialisation du modernisme visuel, à laquelle Vogel et son réseau participent, il convient de s'interroger sur l'intérêt marchand du graphisme de $V U$. L'hypothèse défendue ici est qu'il lui permet de cibler un segment spécifique du public français. Son design est en ce sens un signe de distinction et une stratégie de valorisation d'un genre de presse hybride inédit, dont le succès commercial comme la position dans l'espace des légitimités culturelles restent à déterminer. Il participe pleinement d'une formule médiatique originale qui peut être qualifiée de middlebrow. Ce terme, à l'origine dépréciatif, désigne dans les contextes anglophones une culture commerciale qui prend de l'essor au début $\mathrm{du} \mathrm{XX}^{\mathrm{e}}$ siècle, née de l'industrialisation de la production littéraire, et située entre le "canon » et la culture " authentiquement » populaire ${ }^{49}$. Ses fonctions pédagogiques ou ses usages sociaux ont été envisagés dans une perspective d'inspiration volontiers bourdieusienne : sous cet angle, le middlebrow est une catégorie relative et déterminée par des dynamiques de champ ${ }^{50}$. Elle viserait essentiellement à alimenter la "bonne volonté culturelle $»^{51} \mathrm{de}$ nouvelles et croissantes classes moyennes éduquées ${ }^{52}$. Cependant, les spécialistes 
insistent également sur les spécificités esthétiques du middlebrow. Il a principalement été caractérisé par un syncrétisme des genres littéraires ${ }^{53}$ et par le recours au réalisme, support d'une lecture de plaisir ${ }^{54}$. Appliquées au champ des études visuelles, ces approches éclairent d'une manière nouvelle les mises en page et l'iconographie de $V U$. L'hebdomadaire de Vogel mélange en effet les genres établis sur le plan visuel autant que sur le plan éditorial. Il marie et adapte les différents modèles d'innovation médiatique promus par le réseau d'artistes, d'éditeurs, et de publicitaires qui contribuent à sa production, afin de proposer un produit «moyen» qui s'efforce de définir pour son lectorat les contours de la modernité. $V U$ amorce ainsi un virage dans l'histoire médiatique vers l'épanouissement du format magazine, support privilégié d'une nouvelle culture de masse dotée d'une esthétique propre.

Il est à ce titre symptomatique des métamorphoses de l'édition de presse dans l'entredeux-guerres français. L'accès croissant de nouvelles couches sociales à une consommation culturelle jusque-là réservée aux catégories les plus aisées menace la culture bourgeoise des élites, dont le pouvoir d'influence est remis en question ${ }^{55}$. Selon l'historienne Marjorie Beale, c'est paradoxalement en s'investissant dans le développement des communications de masse, et en s'alliant à des artistes modernes aux discours pourtant contestataires, qu'une partie des classes privilégiées réaffirment en France leur position dominante ${ }^{56}$. Les médias et la publicité sont le terrain sur lequel elles peuvent négocier en leur faveur ces transformations de la société. De plus, au tournant des années 1930, le secteur de la presse est concurrencé par de nouveaux supports d'information. Fragilisé, il doit se renouveler ${ }^{57}$. Pour les éditeurs comme Vogel, s'assurer la fidélité de nouveaux publics n'est donc pas seulement une option : c'est une nécessité. Avant VU, Vogel a ainsi fait sa spécialité de la " démocratisation " de la presse féminine et, plus précisément, de la conquête de nouveaux lectorats au sein de catégories sociales émergentes, telles que les femmes salariées de classe moyenne ${ }^{58}$. Celles-ci constituent un marché prometteur pour les producteurs médiatiques, qui adaptent leur offre en valorisant de nouveaux modèles de féminité partiellement émancipé ${ }^{59}$. Après avoir quitté la direction de son hebdomadaire en 1936, l'éditeur est chargé de moderniser Le Petit Journal, quotidien populaire, et d'étendre son influence auprès des classes moyennes. Il revoit la maquette de l'hebdomadaire de gauche Marianne, qu'il enrichit de rubriques culturelles, puis rejoint le quotidien syndical Messidor, dont il dirige la mise en page ${ }^{60}$. Que ce soit par la vulgarisation des formats luxueux de la presse de mode ou par l'élévation graphique de journaux populaires, la séduction de lectorats intermédiaires représente donc le domaine de prédilection de Vogel. Avec VU, il mobilise le savoir-faire acquis au cours de la première phase de sa carrière, et le réseau professionnel qu'il a constitué, pour les déployer dans le cadre d'une entreprise journalistique davantage politique, qui le mènera à ses projets ultérieurs.

Comme l'indique l'éditorial «Remarques sur un nouveau journal illustré », la formule de VU est d'emblée donnée comme hétéroclite. Elle mélange des aspects conventionnels de la presse d'actualité et des formes novatrices mais populaires. Les publications consacrées au cinéma ou au sport, comme Match L'Intran, inventent en effet depuis plusieurs années des manières originales d'informer visuellement ${ }^{61}$. Liberman effectue d'ailleurs quelques travaux pour l'hebdomadaire de cinéma Pour Vous avant son arrivée à $V U^{62}$. Le caractère composite de $V U$ est particulièrement frappant dans ses pages culturelles et ses choix de feuilletons. Les lecteurs et lectrices de l'hebdomadaire 
peuvent se divertir à la fois de nouvelles de Jack London, d'Alain Saint-Ogan, créateur de la bande dessinée Zig et Puce, de Johnston McCulley, créateur de Zorro, ou d'Horace Walpole, pionnier du roman gothique; de récits policiers de Georges Simenon, des mémoires du Maréchal Joffre, du journal de guerre de Benito Mussolini, d'extraits du Voyage au bout de la nuit de Louis-Ferdinand Céline, de lettres de Marcel Proust et des aventures de Babar l'éléphant, création de Jean de Brunhoff, beau-frère de Vogel. VU alterne des références à la culture savante et à la culture populaire; à la presse d'opinion et à la presse de divertissement. La filiation de la presse féminine, enfin, est indéniable. $V U$ propose par exemple de fréquents hors-série consacrés à la mode, coordonnés par Cosette Vogel de Brunhoff ${ }^{63}$, et trois numéros spéciaux dédiés à la «situation actuelle $»^{64}$ des femmes en France et dans le monde (Fig. 7). Le public féminin joue de toute évidence un rôle majeur dans le projet généraliste de $V U$. Dans le sillage des travaux de Rita Felski sur « le genre de la modernité " ${ }^{65}$, les recherches sur la culture middlebrow ont de fait souligné le poids déterminant de la consommation féminine dans la définition d'une culture "médiane ", voire dans la constitution d'une " conscience de classe moyenne $»^{66}$. Tout comme le mélange des genres journalistiques, le recadrage de préoccupations jugées féminines en sujets d'actualité "grand public » paraît être au fondement de l'ouverture d'un espace médiatique intermédiaire. D'autres périodiques d'actualité photographique, disposant chacun de leurs spécificités, y rejoignent bientôt $V U$, tels que le moins sérieux Voilà en 1931, ou la nouvelle formule de Match, qui étend en 1938 son champ d'intérêt de l'actualité sportive à l'actualité mondiale.

Fig.7. Trois numéros spéciaux consacrés à l'actualité des femmes : VU, 48, 13 février 1929 ; VU, Hors-Série « Femmes, Noël », 9 décembre 1933 ; VU, Hors-Série " Joies et plaisirs des femmes ", 26 octobre 1935. Format : $27 \times 37 \mathrm{~cm}$. Reproduction : Musée Nicéphore Niépce, Ville de Chalon-surSaône.
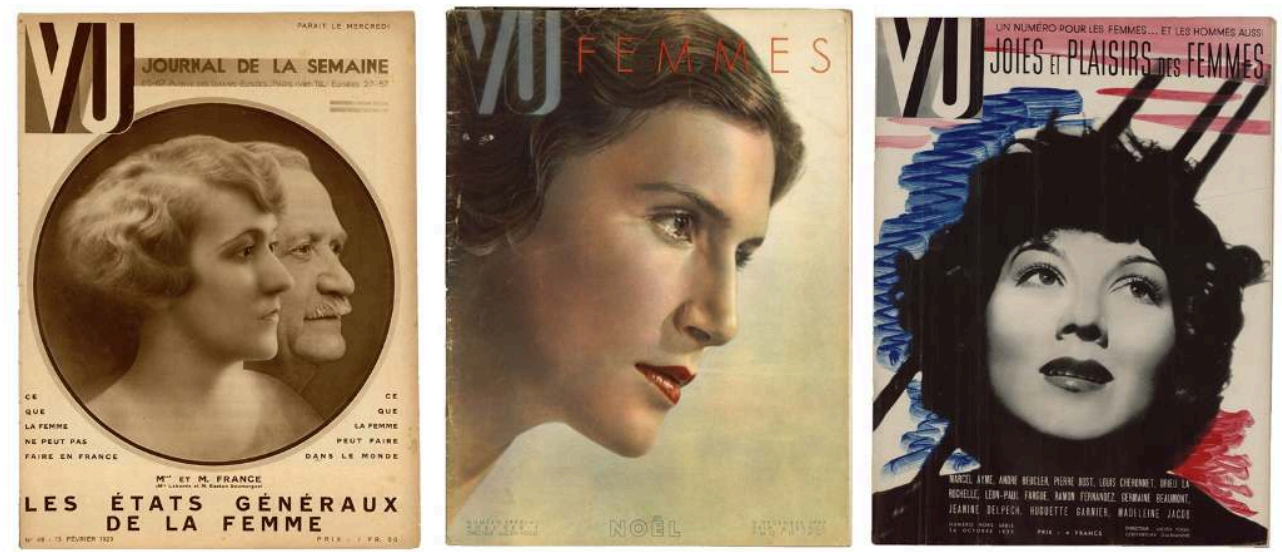

Visuellement, $V U$ ressemble moins aux journaux d'information qui lui sont contemporains qu'aux revues de mode, attentives aux variations de l'actualité vestimentaire et décorative, qui accordent un soin logique à leur identité graphique. Les rubriques et numéros spéciaux de VU consacrés à ces sujets, en particulier, allient typiquement l'épure caractéristique du «style Bauhaus » à des effets de composition traditionnels tirés de l'album, tels que l'usage de marges très larges ou de colonnes symétriques. Les titres sont fréquemment composés en «style Coquemer ", qui consiste à remplacer les lettres majuscules par des minuscules d'un format supérieur (Fig. 8). Il s'agit d'un procédé à la fois moderniste, puisqu'il permet une composition intégralement en « bas de casse », mais également ornemental et donc, à mille lieues du 
fonctionnalisme prôné par Tschichold et ses pairs. De même, dans ses illustrations photographiques, VU parvient à conjuguer le réalisme documentaire du photoreportage avec la recherche formelle, tirant vers l'abstraction, de la Nouvelle objectivité (Fig. 9). Nourrie de l'influence allemande et de celle des arts graphiques français, son identité visuelle évoque à la fois une rupture des traditions éditoriales, la sophistication de l'intelligentsia, et l'imagerie publicitaire familière des grands boulevards parisiens, dominés par le style art-déco de Cassandre, Carlu ou Brodovitch. Lidova collabore d'ailleurs avec ce dernier à la direction artistique des Trois Quartiers ${ }^{67}$, et peut s'inspirer de cette expérience pour son travail à $V U$. Le périodique déploie ainsi un modernisme accessible et adapté au public français.

Fig.8. Un exemple de titraille composée en « style Coquemer » dans VU, 252, 11 janvier 1933, pp. 48-49. Format : $54 \times 37 \mathrm{~cm}$. Reproduction : Musée Nicéphore Niépce, Ville de Chalon-sur-Saône.

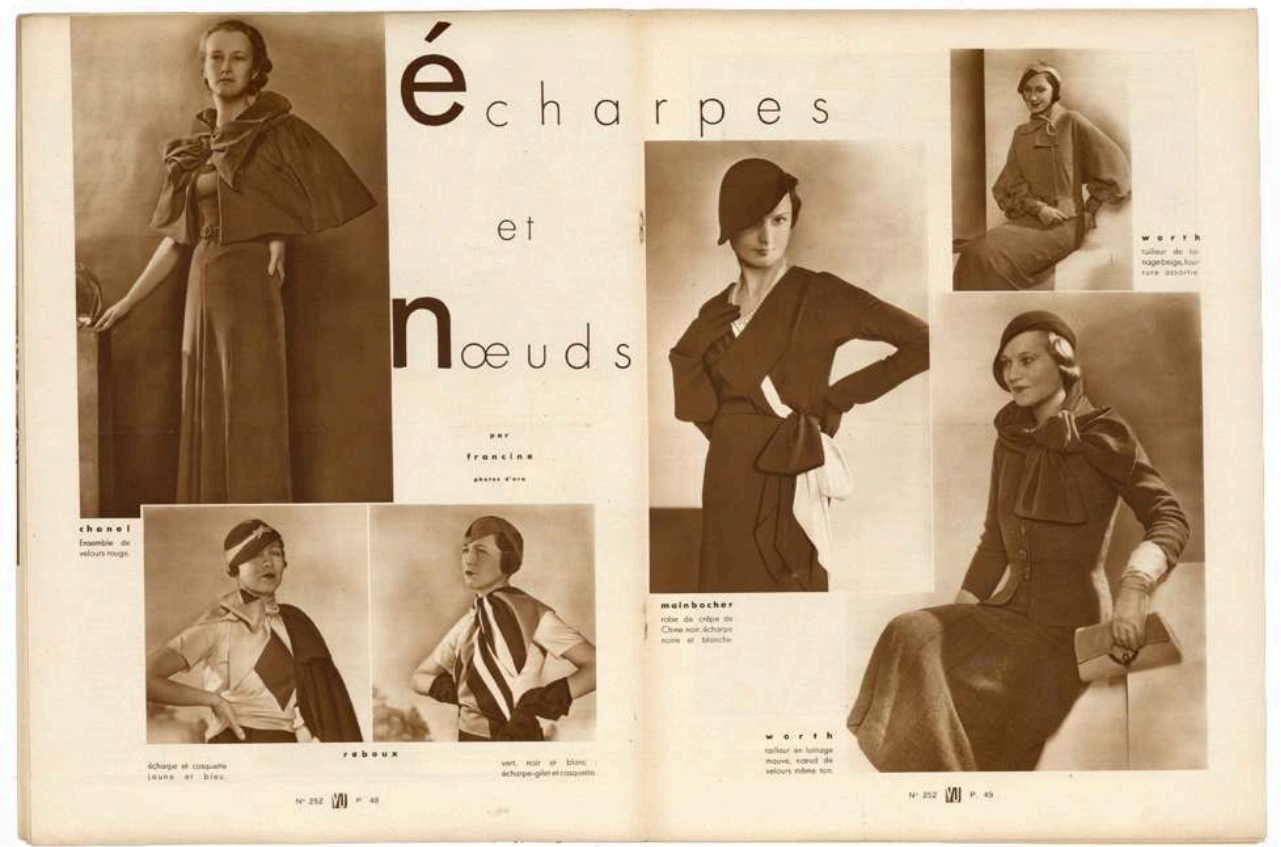


Fig.9. VU, 427, 20 mai 1936, pp. 570-571. Format : $54 \times 37 \mathrm{~cm}$. Reproduction : Musée Nicéphore Niépce, Ville de Chalon-sur-Saône.

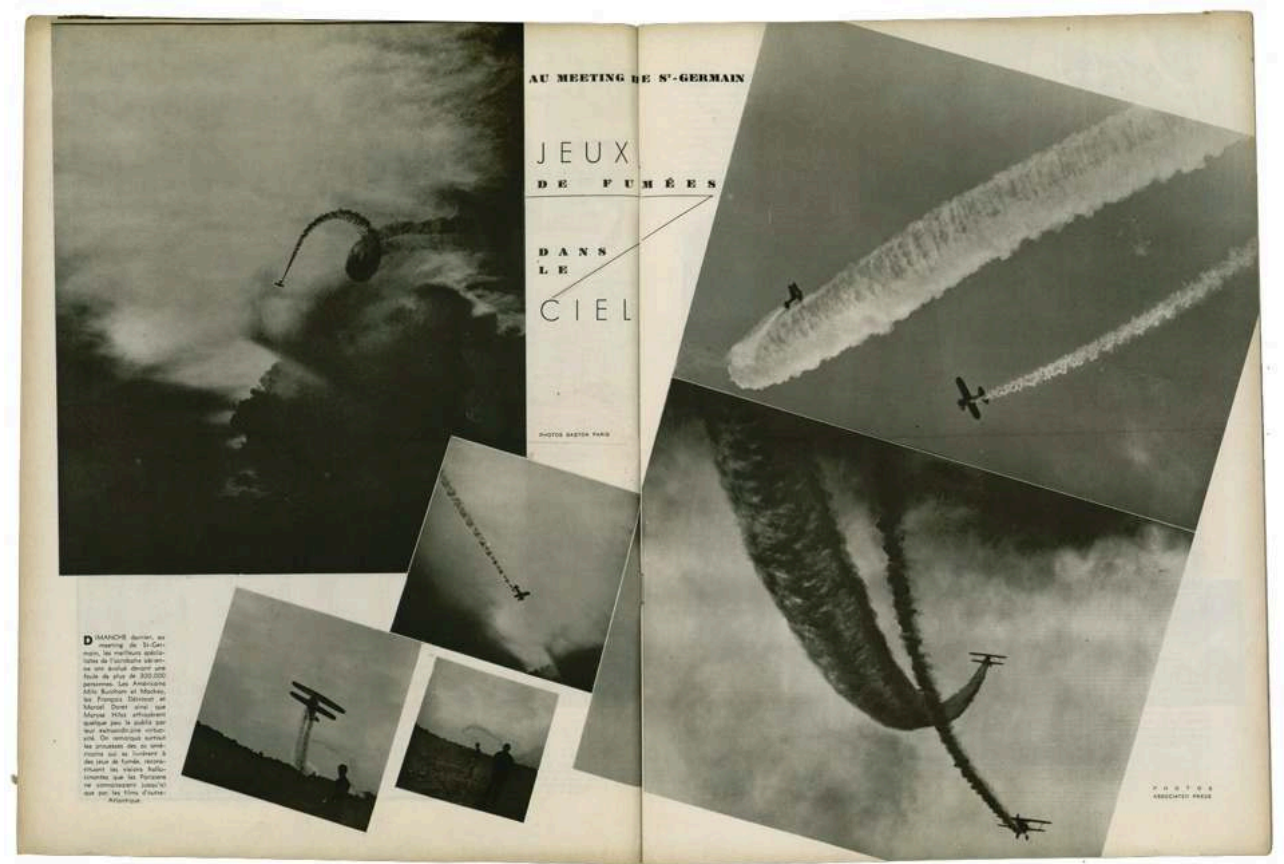

17 Une comparaison peut être établie avec die neue linie, revue lifestyle que Patrick Rössler analyse comme une réponse du marché allemand à l'échec commercial de Vogue ${ }^{68}$. Au pays du «style Bauhaus », la formule empesée que propose encore Condé Nast en 1929 n'est plus suffisamment prestigieuse pour son public-cible. L'esthétique commerciale dérivée du modernisme artistique, en revanche, interpelle les groupes sociaux sensibles à la promesse de radicalité qu'elle représente. Selon Schwartz, ils réunissent essentiellement de jeunes salariée's citadin'e's soucieu'x'ses de la mode et avides de divertissements commerciaux ${ }^{69}$. Ils appartiennent à une nouvelle classe moyenne qui apprécie à la fois la commodité et l'aura anticonformiste des marchandises industrielles épurées, dont le style est parfois qualifié de "bolchéviste ${ }^{70}$. Le rejet de l'ornement dans l'habillement ou la décoration est en effet associé au rejet du prestige bourgeois. Le parti pris esthétique de die neue linie n'est donc pas anodin : il est un moyen pour le mensuel de canaliser un intérêt collectif pour de nouvelles formes de vie. Il est légitime de supposer que $V U$ opère de la même manière. Le contexte médiatique, culturel, social et politique français de la fin des années 1920 diffère certes de la situation allemande. Le conservatisme formel et politique de L'Illustration, par exemple, ne l'empêche pas d'être l'hebdomadaire illustré au tirage le plus important de la période ${ }^{71}$. Cependant, c'est précisément en réaction à ce cadre que peut se comprendre le projet de VU. Il se situe à mi-chemin entre la valorisation culturelle du divertissement et de la création industrielle, et la popularisation de sujets sérieux et de savoirs légitimes, problématique d'actualité et bientôt au cœur de la politique culturelle du Front Populaire $^{72}$. VU propose en effet un contenu d'information proche de celui de L'Illustration, mais le met à disposition pour un prix deux fois inférieur ${ }^{73}$ et l'associe à un positionnement à la fois politique et esthétique radicalement différent. Le graphisme de $V U$, hybride et d'inspiration internationale, peut ainsi être envisagé comme un indice de sa caractérisation sociale. Situé politiquement plus à gauche que L'Illustration, moins populaire que les hebdomadaires thématiques ou satiriques, moins élitiste et 
moins genré que les revues onéreuses telles que Vogue, il paraît conçu sur mesure pour de nouveaux groupes intermédiaires, qui se définissent précisément par leur sentiment de n'appartenir ni à la classe ouvrière, ni à la bourgeoisie ${ }^{74}$. Relativement instruits et susceptibles de remettre partiellement en cause l'ordre social, mais aspirant néanmoins au confort matériel, ils sont en quête de manières de vivre qui leur soient propres et d'une culture médiatique commune.

Les annonces publicitaires de VU confirment et étoffent ce portrait de son lecteur et de sa lectrice idéaux. Ce sont avant tout des citadins, clients potentiels du restaurant Viking, dans le quartier cosmopolite de Montparnasse; et de magasins d'ameublement du type de celui de Marc Chalier, maitre du luminaire moderne, sur le boulevard SaintGermain. Ils consomment des liqueurs de luxe, possèdent un appareil photographique, un poste TSF dernier cri, une voiture (Fig. 10), une yaourtière et une cocotte en Pyrex. Ils consultent le Bottin Mondain, fréquentent les plages du Touquet ou de Biarritz avant l'instauration des congés payés ${ }^{75}$ et envisagent de visiter la Suisse (Fig. 11) ou l'Égypte. Ils correspondent au prototype des jeunes cadres parisiens, clients du Salon des Arts Ménagers, dont les goûts préfigurent de nouveaux modes de consommation ${ }^{76}$. Ils se situent dans le haut de la classe moyenne, à une époque où, malgré l'accroissement des salaires en cours, l'achat d'une machine à laver demeure un luxe inaccessible pour beaucoup, et les voyages un signe de niveau de vie élevé ${ }^{77}$. On peut toutefois imaginer que ce profil-type ne reflète pas l'ensemble du lectorat de l'hebdomadaire, et que l'opulence relative de ses pages publicitaires serve aussi une fonction de prestige. On y trouve occasionnellement des annonces pour La Belle Jardinière, grand magasin de confection à prix fixes dévolu à une clientèle davantage populaire; pour les établissements Nicolas, innovants sur le plan des techniques de vente et de publicité, qui bradent leurs vins de qualité pour toucher de nouveaux publics ${ }^{78}$; ou encore pour les voitures économiques Simca qui favorisent la démocratisation de l'automobile ${ }^{79}$.

Fig.10. VU, Hors-Série «Femmes, Noël », 9 décembre 1933, pp. 2-3. Format : $54 \times 37 \mathrm{~cm}$. Reproduction : Musée Nicéphore Niépce, Ville de Chalon-sur-Saône.

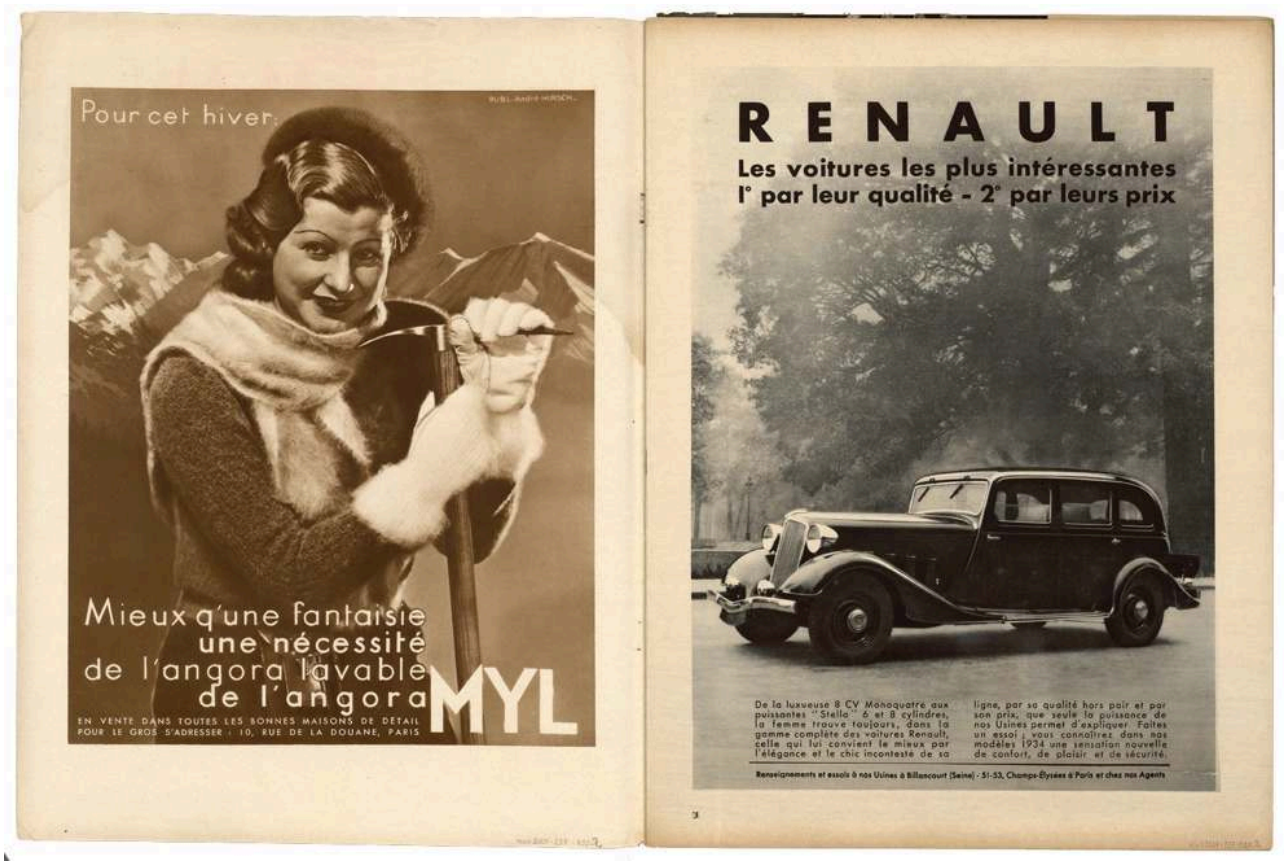


Fig.11. VU, Hors-Série "Paris en fête", 16 juin 1934, pp. 6-7. Format : $54 \times 37 \mathrm{~cm}$. Reproduction : Musée Nicéphore Niépce, Ville de Chalon-sur-Saône.

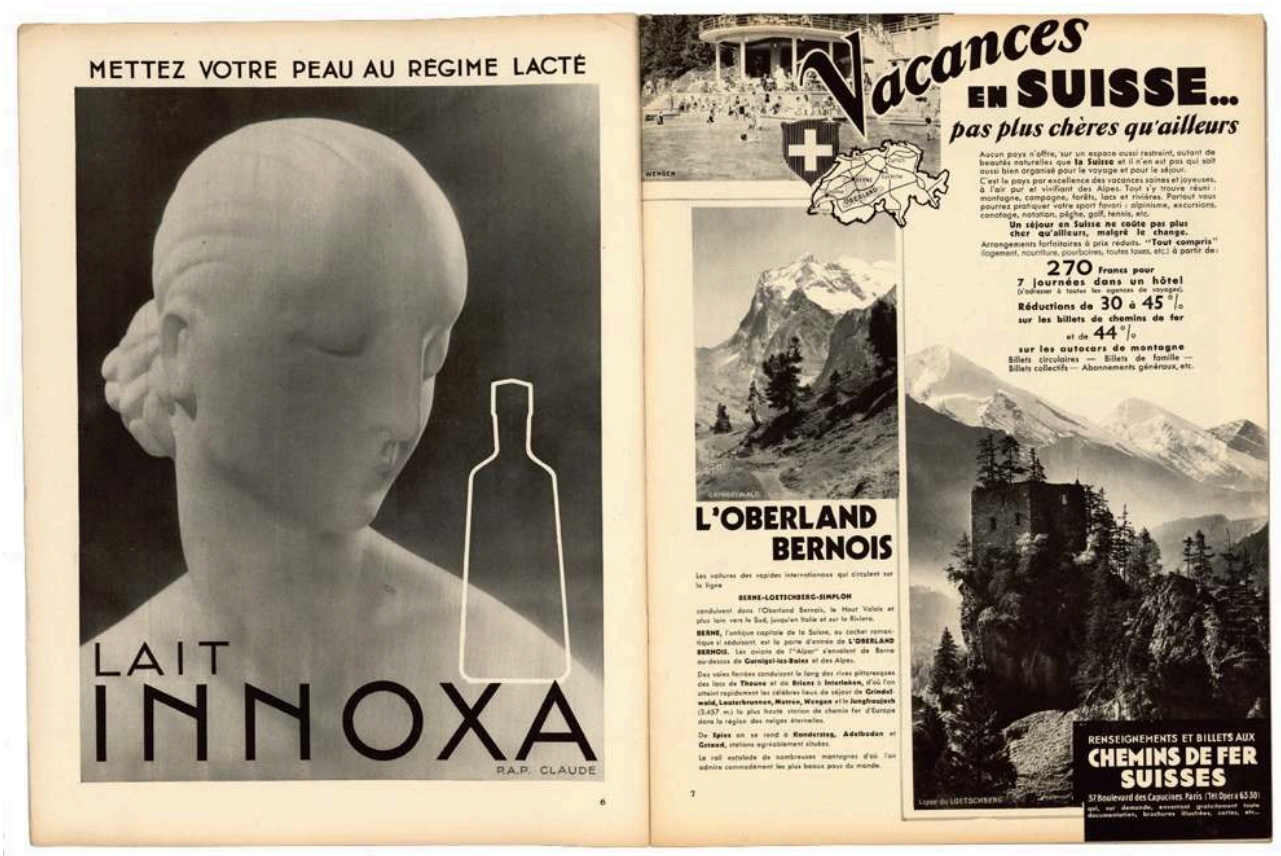

19 Dès son premier éditorial, VU promet par ailleurs d'accorder à la publicité «la place qu'elle occupe dans la vie moderne $»^{80}$ et en fait, avec le "reportage illustré d'informations mondiales", l'une des clés de voûte de la «formule neuve " qu'il propose. Aux côtés des rubriques «Explorations, Exploits sportifs, Théâtre, Cinéma, Art, Mode ", la consommation est présentée comme un aspect fondamental de la modernité portée par l'hebdomadaire. $V U$ est en outre impliqué dans une manifestation au caractère précurseur ${ }^{81}$ qui œuvre à l'ouverture des marchés français : Le Train de France, défini par ses créateurs comme « une exposition mobile de grande propagande destinée non seulement à réaliser la meilleure éducation du consommateur, mais encore la création de la demande la plus fructueuse pour le commerçant», un «groupement sélectionné des produits et de la publicité de 600 à 700 exposants (...) choisis dans tout ce qui touche au grand domaine du Foyer et de l'Art Ménager ${ }^{82}$. Destinée à être déployée dans une cinquantaine de villes françaises au cours de l'année 1929, elle est présentée en avant-première au Salon du Foyer à Versailles en 1928. Ces événements sont organisés par un groupe d'industriels et par Jules-Louis Breton, créateur de Salon des Arts Ménagers. Le Salon du Foyer expose également les travaux de plusieurs architectes de l'UAM ${ }^{83}$. Charles Peignot préside quant à lui la Section «Bureau Moderne, Papeterie, Librairie» du Train de France, au sein de laquelle sont présentés la revue $A M G$ et les produits de la Fonderie D\&P. VU partage son stand et y installe un salon de lecture ${ }^{84}$. Vogel cherche donc, dès la première année de la publication de son hebdomadaire, à l'associer à un effort de modernisation des pratiques de consommation et à conquérir son lectorat jusque dans les zones géographiques que la distribution et la publicité peinent encore à décloisonner ${ }^{85}$. Quels que soient leurs revenus, les lecteurs et lectrices convoités par VU peuvent donc être définis avant tout par leur ouverture aux principes de confort et de délassement, présentés dans le périodique et via ses activités promotionnelles comme les conditions d'accès - monnayables - à un style de vie moderne. 
$20 \mathrm{VU}$ propose en somme une formule médiatique innovante et définie par un contenu éditorial, photographique et typographique qui se joue des hiérarchies culturelles et prône le mélange des formes de communication. "Conçu dans un esprit nouveau et réalisé par des moyens nouveaux $»^{86}$, il promeut une modernité politique et culturelle rendue accessible à son lectorat par la consommation des images et des objets qui l'incarnent. Il revêt à ce titre un rôle prescripteur d'éducation au goût moderne, caractéristique de la culture médiatique middlebrow des années 1930. Aux États-Unis, les rubriques de Life consacrées à l'architecture et à la décoration endossent une fonction similaire de popularisation de l'art moderne ${ }^{87}$. Au Royaume-Uni, des architectes réunis autour de la revue The Architectural Review et des entreprises comme Penguin et la BBC forment un réseau qui, à l'instar de celui de $V U$, cible une portion spécifique des classes moyennes pour cultiver chez elles un intérêt pour le design moderniste ${ }^{88}$. Après la guerre, la modernité visuelle apprivoisée de $V U$ envahit la presse de mode américaine en mutation. Harper's Bazaar et Vogue, dont les liens avec VU ont été établis, en deviennent les principaux représentants. L'hebdomadaire est donc un des acteurs qui rendent possible l'essor progressif du «style international » en design, constitutif de l'esthétique industrielle et publicitaire des années 1950 et 1960 et dérivé du modernisme porté par le Bauhaus. Au début des années 1930, VU n'est plus un journal illustré mais bel et bien un magazine, nouveau média moderne caractérisé par sa visualité et par sa capacité à regrouper une communauté de lecteurs et de lectrices autour d'une esthétique de vie commune ${ }^{89}$. Il participe ainsi à l'élaboration de la culture visuelle « moyenne » du média magazine.

\section{Conclusion}

21 Tout en prétendant s'adresser à un public large, VU s'associe à l'avant-garde artistique et adapte ses innovations formelles afin de servir un positionnement commercial original. Bien ancrée dans un réseau international de producteurs médiatiques et de publicitaires, la publication synthétise les savoir-faire les plus innovants pour séduire un segment précis des classes moyennes françaises, mettant en œuvre ce que l'on pourrait décrire aujourd'hui comme une stratégie marketing complète. Elle initie son lectorat à une nouvelle conception du quotidien, qui valorise le divertissement autant que la réflexion sérieuse, et contribue à établir la consommation au cœur des pratiques culturelles. Elle annonce l'avènement d'un genre de presse nouveau, le magazine, et d'une culture visuelle moderne qui lui est intimement liée. L'esthétique introduite dans les médias français par $V U$, qui dilue la radicalité du modernisme artistique dans une modernité davantage ordinaire, héritée de la publicité ou des hebdomadaires spécialisés, peut être interprétée comme un équivalent de la catégorie middlebrow sur le terrain de la communication graphique et du design. VU peut donc être compris comme un effort et une réussite partielle de «moyennisation » culturelle. Partielle seulement, car il ne rencontre pas le succès peut-être escompté par Vogel. Selon Françoise Denoyelle, il peine à séduire à la fois la petite-bourgeoisie, pour qui il est trop teinté politiquement, et les lecteurice-s de gauche qui lui préfèrent le moins modéré Regards ${ }^{90}$. Enfermé dans la niche qu'il s'est pourtant, semble-t-il, appliqué à définir, $V U$ ne devient jamais le grand journal qu'il ambitionne d'être et qu'est immédiatement Life aux États-Unis. Celui-ci est pourtant conçu au milieu des années 1930 avec l'implication de l'agence Dorland ${ }^{91}$ et sur le 
modèle de VU. Clare Boothe, rédactrice en chef de Vanity Fair, suggère en effet dès 1931 à Condé Nast de mettre sur pied un hebdomadaire équivalent pour le marché américain, avant de faire part du projet à Henry Luce qui le mène à bien en $1936^{92}$. Fragilisé économiquement, c'est lorsque Vogel cherche à affirmer la ligne politique de son hebdomadaire qu'il perd la confiance de ses annonceurs et est contraint, en 1936, à revendre ses parts de la Société des Illustrés Français ${ }^{93}$. La réorientation éditoriale de $V U$, davantage à droite, qui en découle, ne fait rien pour le succès du titre dont l'audience périclite et qui disparaît définitivement en 1940. Ce projet journalistique inventif a toutefois ouvert la voie à l'explosion de l'offre magazine sur le marché de la presse française, qui aura lieu une vingtaine d'années plus tard.

\section{BIBLIOGRAPHIE}

$2^{e}$ Salon du Foyer : catalogue officiel, Paris, S. A. M., 1928.

Fonderie Deberny et Peignot : coupures de presse, Paris, Bibliothèque Forney, Archives Peignot, Fonds Charles Peignot, Boîte VIII, 2.

Les Trains de France, expositions mobiles de grande envergure, Paris, Trains de France, 1928.

Les Trains de France, expositions mobiles de grande envergure : catalogue officiel, Paris, Trains de France, 1928.

Spécimen général, Paris, Deberny \& Peignot,1926.

Spécimen général, Paris, Deberny \& Peignot,1935.

James Sloan Allen, The Romance of Commerce and Culture: Capitalism, Modernism, and the ChicagoAspen Crusade for Cultural Reform, Boulder, University Press of Colorado, 2002.

Jeremy Aynsley, Graphic Design in Germany: 1890-1945, Berkeley, University of California Press, 2000.

Marjorie Anne Beale, The Modernist Enterprise: French Elites and the Threat of Modernity, 1900-1940, Palo Alto, Stanford University Press, 1999.

Claude Bellanger et al. (dir.), Histoire générale de la presse française, Tome III, 1871-1940, Paris, Presses Universitaires de France, 1972.

Serge Berstein, « Les classes moyennes devant l'histoire », Vingtième Siècle. Revue d'histoire, 37, 1, 1993, pp. 3-12.

Amy L. Blair, Reading up: middle-class readers and the culture of success in the early twentieth-century United States, Philadelphia, Temple University Press, 2012.

Claire Blandin (dir.), Manuel d'analyse de la presse magazine, Malakoff, Armand Colin, 2018.

Marshall Blonsky, « Alexander Liberman », BOMB, 16, 1986, pp. 18-25.

Pierre Bourdieu, La distinction : critique sociale du jugement, Paris, Minuit, 1979.

Christopher Burke, Active literature: Jan Tschichold and new typography, London, Hyphen, 2007. 
Marie-Emmanuelle Chessel, La publicité : naissance d'une profession, 1900-1940, Paris, CNRS Éditions, 1998.

Marie-Emmanuelle Chessel, Histoire de la consommation, Paris, La Découverte, 2012.

Jean-Claude Daumas, La révolution matérielle : Une histoire de la consommation, Paris, Flammarion, 2018.

Christian Delporte, Claire Blandin et François Robinet, Histoire de la presse en France : $\mathrm{XX}^{e}-\mathrm{XXI}^{e}$ siècles, Paris, Armand Colin, 2016.

Françoise Denoyelle, La lumière de Paris. 2 : Les usages de la photographie, 1919-1939, Paris, L'Harmattan, 1997.

Alexandre Dumas de Rauly et Michel Wlassikoff, Futura : une gloire typographique, Paris, Norma, 2011.

Rita Felski, The Gender of Modernity, Cambridge (MA), Harvard University Press, 1995.

Gilles Feyel, « Naissance, constitution progressive et épanouissement d'un genre de presse aux limites floues : le magazine », Réseaux, 1, 105, 2001, pp. 19-51.

Michel Frizot et Cédric de Veigy, Vu : le magazine photographique 1928-1940, Paris, La Martinière, 2009.

Kristof van Gansen, «"Plaidoyer pour le graphiste" : Arts et métiers graphiques and the French Typographer as an Artist and Craftsman », TMG Journal for Media History, 19, 2, 2016, pp. 1-15.

Alexie Geers, Le sourire et le tablier. La construction médiatique du féminin dans Marie-Claire de 1937 à nos jours, Thèse de doctorat en Histoire sous la direction d'André Gunthert et de Rose-Marie Lagrave, EHESS, Paris, 2016.

Thierry Gervais, «L'invention du magazine : La photographie mise en page dans "La Vie au grand air" (1898-1914) », Études photographiques, 20, 2007.

Thierry Gervais avec la collaboration de Gaëlle Morel, La fabrique de l'information visuelle : photographies et magazines d'actualité, Paris, Textuel, 2015.

Elizabeth Hennessy et Ian Keil, « Dorland: Its Origins and Growth to 1939 », European Journal of Marketing, 22, 8, 1988, pp. 49-67.

Diana Holmes, « Introduction: European Middlebrow », Belphégor, 15, 2, 2017.

Diana Holmes, « Une littérature illégitime - le "middlebrow” ", Belphégor, 17, 1, 2019.

Nicola Humble, The Feminine Middlebrow Novel, 1920s to 1950s: Class, Domesticity, and Bohemianism, Oxford, Oxford University Press, 2001.

Nicola Humble, « Sitting Forward or Sitting Back: Highbrow v. Middlebrow Reading », Modernist Cultures, 2011, 6, 1, pp. 41-59.

Béatrice Joyeux-Prunel, Les avant-gardes artistiques 1918-1945 : une histoire transnationale, Paris, Gallimard, 2017.

Dominique Kalifa, La culture de masse en France, Paris, La Découverte, 2001.

Dodie Kazanjian et Calvin Tomkins, Alex: the life of Alexander Liberman, New York, A. A. Knopf, 1993.

Jessica Kelly, «'To Fan the Ardour of the Layman': The Architectural Review, The MARS Group and the Cultivation of Middle Class Audiences for Modernism in Britain, 1933-1940 ", Journal of Design History, 29, 4, 2016, pp. 350-365. 
Sophie Kurkdjian, Lucien Vogel et Michel de Brunhoff, parcours croisés de deux éditeurs de presse illustrée au XXe siècle, Clermont-Ferrand, Institut Universitaire Varenne, 2014.

Sophie Kurkdjian, « L'édition de presse selon Lucien Vogel : l'inventivité dans la diversité », Revue de la BNF, 49, 1, 2015, pp. 60-71.

Danielle Leenaerts, Petite histoire du magazine Vu (1928 - 1940) : entre photographie d'information et photographie d'art, Bruxelles, PIE Lang, 2010.

Irène Lidova, Ma vie avec la danse, Paris, Plume, 1992.

Jan Logemann, Engineered to Sell: European Émigrés and the Making of Consumer Capitalism, Chicago, University of Chicago Press, 2019.

Ellen Lupton, Herbert Bayer: Inspiration and Process in Design, New York, Princeton Architectural Press, 2020.

Susanne Marten-Finnis et Michael Nagel (dir.), Die Pressa: Internationale Presseausstellung Köln 1928 und der jüdische Beitrag zum modernen Journalismus / The Pressa: International Press Exhibition, Cologne, 1928 and the Jewish Contribution to Modern Journalism, Bremen, Edition Lumière, 2012.

Julia Meer, Neuer Blick auf die Neue Typographie: die Rezeption der Avantgarde in der Fachwelt der 1920er Jahre, Bielefeld, Transcript, 2015.

Jörg Meißner, «Quand l'art moderne devient commercial », Vingtième Siècle. Revue d'histoire, 101, 1, 2008, pp. 27-48.

Louis Merlin, J'en ai vu des choses !, Paris, R. Julliard, 1962.

Pascal Ory, La belle illusion : culture et politique sous le signe du Front populaire, 1935-1938, Paris, CNRS Éditions, 2016.

Charles Peignot, « Les Peignot: Georges, Charles », Communication \& Langages, 59, 1, 1984, pp. 61-85.

Janice A. Radway, A Feeling for Books: the Book-of-the-Month Club, Literary Taste, and Middle-Class Desire, Chapel Hill, University of North Carolina Press, 1997.

Susan Ronald, Condé Nast: The Man and His Empire - A Biography, New York, St. Martin's Press, 2019.

Patrick Rössler, Die neue Linie 1929-1943: das Bauhaus am Kiosk, Bielefeld, Kerber, 2007.

Patrick Rössler, « Vielfalt in der Gleichschaltung - die »domestizierte Moderne« am Kiosk: Eine Lifestyle-Illustrierte zwischen Bauhaus-Avantgarde und NS-Propaganda: >die neue linier 1929-1943 ", Jahrbuch für Kommunikationsgeschichte, 9, 2007, pp. 150-195.

Joan Shelley Rubin, The Making of Middlebrow Culture, Chapel Hill, University of North Carolina Press, 1992.

Frederic J. Schwartz, « Utopia for sale: the Bauhaus and Weimar Germany's consumer culture », dans Kathleen James (dir.), Bauhaus Culture: from Weimar to the Cold War, Minneapolis, University of Minnesota Press, 2006.

Martine Segalen, « The Salon des Arts Ménagers, 1923-1983: A French Effort to Instil the Virtues of Home and the Norms of Good Taste », Journal of Design History, 7, 4, 1994, pp. 267-275.

Jan Tschichold, La Nouvelle Typographie, traduit par Philippe Buschinger et Françoise Buschinger, Montreuil / Genève, Entremonde, 2016. 
Sheila Webb, « Art Commentary for the Middlebrow: Promoting Modernism \& Modern Art through Popular Culture-How Life Magazine Brought "The New" into Middle-Class Homes ", American Journalism, 27, 3, 2010, pp. 115-150.

\section{NOTES}

1. Michel Frizot et Cédric de Veigy, Vu : le magazine photographique 1928-1940, Paris, La Martinière, 2009.

2. Sophie Kurkdjian, Lucien Vogel et Michel de Brunhoff, parcours croisés de deux éditeurs de presse illustrée au XXe siècle, Clermont-Ferrand, Institut Universitaire Varenne, 2014.

3. « Remarques sur un nouveau journal illustré », VU, 1, 21 mars 1928, pp. 11-12.

4. Ibid., p. 11 .

5. Vogel voyage à Berlin en 1927 pour visiter l'Ullstein-Verlag, plus grande entreprise de presse allemande et éditrice de la Berliner Illustrirte Zeitung. Voir Sophie Kurkdjian, op. cit., p. 375. L'imprimeur Paul August Ringier qui édite la Schweizer Illustrierte Zeitung et l'Unterhaltungsblätter est quant à lui actionnaire de la société qui édite VU. Voir Danielle Leenaerts, Petite histoire du magazine $\mathrm{Vu}$ (1928-1940) : entre photographie d'information et photographie d'art, Bruxelles, PIE Lang, 2010, p. 26.

6. Claude Bellanger et al. (dir.), Histoire générale de la presse française, Tome III, 1871-1940, Paris, Presses Universitaires de France, 1972, p. 120.

7. Irène Lidova, Ma vie avec la danse, Paris, Plume, 1992, p. 22.

8. Marshall Blonsky, « Alexander Liberman », BOMB, 16, 1986, pp. 18-25, p. 18.

9. Dodie Kazanjian et Calvin Tomkins, Alex: the life of Alexander Liberman, New York, A. A. Knopf, 1993 , p. 53.

10. Françoise Denoyelle, La lumière de Paris. 2: Les usages de la photographie, 1919-1939, Paris, L'Harmattan, 1997, p. 133 ; Jean-Luc Froissart, L'or, l'âme et les cendres du plomb : l'épopée des Peignot, 1815-1983, Paris, J.-L. Froissart, 2004.

11. Kristof van Gansen, " "Plaidoyer pour le graphiste": Arts et métiers graphiques and the French Typographer as an Artist and Craftsman », TMG Journal for Media History, 19, 2, 2016, pp. 1-15.

12. Il met en page et illustre l'article-manifeste de l'écrivain Pierre Mac Orlan, "Graphismes », Arts et métiers graphiques, 11, 1929, pp. 645-652.

13. Charles Peignot, «Les Peignot: Georges, Charles », Communication \& Langages, 59, 1, 1984, pp. 61-85, p. 75.

14. Jan Tschichold, «Qu'est-ce que la nouvelle typographie et que veut-elle?», Arts et métiers graphiques, 19, 1930, pp. 46-51.

15. Jan Tschichold, La Nouvelle Typographie, traduit par Philippe Buschinger et Françoise Buschinger, Montreuil / Genève, Entremonde, 2016.

16. László Moholy-Nagy, "The New Typography ", traduit par Sibyl Moholy-Nagy, dans Richard Kostelanetz (dir.), Moholy-Nagy, Londres, A. Lane, 1974, pp. 75-76.

17. László Moholy-Nagy, Peinture photographie film et autres écrits sur la photographie, traduit par Gérard Dallez, Jean Kempf et Catherine Wermester, Nîmes, Jacqueline Chambon, 1993, pp. 103-105.

18. Alexandre Dumas de Rauly et Michel Wlassikoff, Futura : une gloire typographique, Paris, Norma, 2011, p. 67.

19. «Exposition internationale de la Presse à Cologne ", VU, 18, 18 juillet 1928, p. 436 ; Susanne Marten-Finnis et Michael Nagel (eds.), Die Pressa: Internationale Presseausstellung Köln 1928 und der jüdische Beitrag zum modernen Journalismus / The Pressa: International Press Exhibition, Cologne, 1928 and the Jewish Contribution to Modern Journalism, Bremen, Edition Lumière, 2012. 
20. Jeremy Aynsley, Graphic design in Germany 1890-1945, London, Thames \& Hudson, 2000, p. 147. 21. Christopher Burke, Active literature: Jan Tschichold and new typography, London, Hyphen, 2007, p. 57.

22. Sophie Kurkdjian, op. cit., p. 369.

23. Arts et métiers graphiques, Statuts, Paris, Les Annonces de la Seine, 1935.

24. Le tirage de $V U$ est estimé en moyenne entre 50000 et 100000 exemplaires. En 1934, il est tiré à 150000 exemplaires, selon les archives de la préfecture de police de Paris. Voir Sophie Kurkdjian, op. cit., p. 369. Le numéro 192 du 18 novembre 1931, consacré à l'URSS et d'une épaisseur inhabituelle de 200 pages, l'aurait été à 500000 exemplaires selon Claude Bellanger et al. (dir.), op. cit., p. 598. Un courrier de Charles Peignot évoque un tirage de 3000 exemplaires pour AMG. Voir Kristof Van Gansen, op. cit., p. 5.

25. Jean-Luc Froissart, op. cit., p. 309.

26. Sophie Kurkdjian, «L'édition de presse selon Lucien Vogel : l'inventivité dans la diversité », Revue de la BNF, 49, 1, 2015, pp. 60-71, p. 64.

27. Sophie Kurkdjian, Ibid.

28. Elizabeth Hennessy et Ian Keil, « Dorland: Its Origins and Growth to 1939 », European Journal of Marketing, 22, 8, 1988, pp. 49-67, p. 52.

29. Louis Merlin, J'en ai vu des choses !, Paris, R. Julliard, 1962, p. 244.

30. Annuaire de la presse française et étrangère et du monde politique, Paris, 1928, p. 361 ; Annuaire de la presse française et étrangère et du monde politique, Paris, 1931, p. 299. Le contrat avec l'agence Dorland est cité dans l'acte de création de la Société des Illustrés Français, mentionné dans Danielle Leenaerts, loc. cit.

31. Annuaire de la presse française et étrangère et du monde politique (1928), loc. cit.

32. Marshall Blonsky, op. cit., p. 18.

33. Annuaire de la presse française et étrangère et du monde politique, Paris, 1935, pp. 285, 1801.

34. Jan Logemann, Engineered to Sell: European Émigrés and the Making of Consumer Capitalism, Chicago, University of Chicago Press, 2019.

35. Susan Ronald, Condé Nast: The Man and His Empire - A Biography, New York, St. Martin's Press, 2019, ch. 23.

36. Certains récits attribuent à Bayer la direction artistique du Vogue allemand, que lui-même revendique, par exemple dans un entretien du 3 octobre 1981 avec les Archives of American Art, Smithsonian Institution. Cette version n'est pas corroborée par les biographes de Condé Nast qui ne le mentionnent pas et insistent au contraire sur le rôle capital d'Agha dans l'histoire du magazine. Voir Caroline Seebohm, The Man who was Vogue: The Life and Times of Condé Nast, New York, Viking Press, 1982, pp. 229-235 ; Susan Ronald, op. cit., ch. 12. Il est possible, au contraire, que Bayer soit recruté par Agha puis le remplace à Dorland. On sait en effet qu'en 1928, il y occupe d'abord un simple poste de créatif. Voir Jörg Meißner, "Quand l'art moderne devient commercial ", Vingtième Siècle. Revue d'histoire, 101, 1, 2008, pp. 27-48, p. 37. Il est envisageable également, Vogue allemand et Dorland partageant les mêmes locaux, que les fonctions aient été indistinctes.

37. Jörg Meißner, op. cit., p. 38.

38. Patrick Rössler, «Vielfalt in der Gleichschaltung - die »domestizierte Moderne» am Kiosk: Eine Lifestyle-Illustrierte zwischen Bauhaus-Avantgarde und NS-Propaganda: >die neue linier 1929-1943 ", Jahrbuch für Kommunikationsgeschichte, 9, 2007, pp. 150-195.

39. Patrick Rössler, op. cit., p. 160.

40. Ellen Lupton, Herbert Bayer: Inspiration and Process in Design, New York, Princeton Architectural Press, 2020.

41. James Sloan Allen, The Romance of Commerce and Culture: Capitalism, Modernism, and the ChicagoAspen Crusade for Cultural Reform, Boulder, University Press of Colorado, 2002, pp. 29-30 ; 35-77.

42. Dodie Kazanjian et Calvin Tomkins, op. cit., pp. 106-107. 
43. Il devient en 1962 directeur éditorial du groupe Condé Nast, fonction qu'il occupe jusqu'en 1994. À propos des parcours croisés d'Alexey Brodovitch et d'Alexander Liberman, et de leurs contributions à l'histoire de la presse de mode, voir Alice Morin, Au cœur des magazines: de collaborations en négociations, le système des images de mode américaines (années 1960-années 1980), thèse de doctorat en Civilisation américaine sous la direction d'Hélène Le Dantec-Lowry, Paris, Sorbonne Paris-Cité, 2018.

44. Jan Logemann, op. cit., pp. 131-192 ; James Sloan Allen, op. cit., pp. 3-34.

45. Frederic J. Schwartz, «Utopia for sale: the Bauhaus and Weimar Germany's consumer culture ", dans Kathleen James (dir.), Bauhaus Culture: from Weimar to the Cold War, Minneapolis, University of Minnesota Press, 2006, pp. 115-138, p. 112.

46. Julia Meer, Neuer Blick auf die Neue Typographie: die Rezeption der Avantgarde in der Fachwelt der 1920er Jahre, Bielefeld, Transcript, 2015.

47. Marie-Emmanuelle Chessel, La publicité: naissance d'une profession, 1900-1940, Paris, CNRS Éditions, 1998, pp. 131-140.

48. Martine Segalen, "The Salon des Arts Ménagers, 1923-1983: A French Effort to Instil the Virtues of Home and the Norms of Good Taste », Journal of Design History, 1994, vol. 7, n 4, pp. 267-275, p. 73.

49. Joan Shelley Rubin, The Making of Middlebrow Culture, Chapel Hill, University of North Carolina Press, 1992, pp. xi-xiv.

50. Amy L. Blair, Reading up: Middle-class Readers and the Culture of Success in the Early TwentiethCentury United States, Philadelphia, Temple University Press, 2012 ; Janice A. Radway, A Feeling for Books: the Book-of-the-Month Club, Literary Taste, and Middle-Class Desire, Chapel Hill, University of North Carolina Press, 1997.

51. Pierre Bourdieu, La distinction : critique sociale du jugement, Paris, Minuit, 1979, pp. 365-431.

52. Diana Holmes, «Introduction: European Middlebrow », Belphégor, 15, 2, 2017, p. 3.

53. Nicola Humble, The Feminine Middlebrow Novel, 1920s to 1950s: Class, Domesticity, and Bohemianism, Oxford, Oxford University Press, 2001, pp. 4-5 ; Diana Holmes, loc. cit.

54. Nicola Humble, "Sitting Forward or Sitting Back: Highbrow v. Middlebrow Reading", Modernist Cultures, 6, 1, 2011, pp. 41-59 ; Diana Holmes, loc. cit ; Janice A. Radway, op. cit.

55. Dominique Kalifa, La culture de masse en France, Paris, La Découverte, 2001, p. 110 ; Marjorie Anne Beale, The Modernist Enterprise: French Elites and the Threat of Modernity, 1900-1940, Palo Alto, Stanford University Press, 1999, p. 6.

56. Marjorie Anne Beale, op. cit., pp. 11-47.

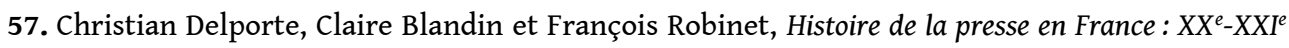
siècles, Paris, Armand Colin, 2016, pp. 92-122.

58. Sophie Kurkdjian, op. cit. (2015), p. 64 ; Françoise Battagliola, Histoire du travail des femmes, Paris, La Découverte, 2008, pp. 57-70.

59. Elizabeth Otto et Vanessa Rocco (dir.), The new woman international: representations in photography and film from the 1870s through the 1960s, Ann Arbor, The University of Michigan Press, 2012 ; Alexie Geers, Le sourire et le tablier. La construction médiatique du féminin dans Marie-Claire de 1937 à nos jours, Thèse de doctorat en Histoire sous la direction d'André Gunthert et de Rose-Marie Lagrave, EHESS, Paris, 2016.

60. Sophie Kurkdjian, op. cit. (2015), pp. 67-69.

61. Thierry Gervais, «L'invention du magazine : La photographie mise en page dans "La Vie au grand air" (1898-1914) », Études photographiques, 20, 2007.

62. Dodie Kazanjian et Calvin Tomkins, op. cit., p. 52.

63. Sophie Kurkdjian, op. cit. (2014), p. 400.

64. Louis Martin-Chauffier, «Présentation », VU, Hors-Série « Femmes, Noël », 9 décembre 1933, p. 19.

65. Rita Felski, The Gender of Modernity, Cambridge (MA), Harvard University Press, 1995. 
66. Janice Radway, « On the Gender of the Middlebrow Consumer and the Threat of the Culturally Fraudulent Female », South Atlantic Quarterly, 1994, pp. 871-893 ; Nicola Humble, op. cit. (2001), pp. 57-107.

67. Dodie Kazanjian et Calvin Tomkins, op. cit., p. 106.

68. Patrick Rössler, op. cit., p. 158.

69. Frederic J. Schwartz, op. cit., pp. 132-133.

70. Frederic J. Schwartz, op. cit., p. 132.

71. Après un pic à 300000 exemplaires en 1915, suivi d'une chute rapide jusqu'à 98000 exemplaires en 1921, les tirages de L'Illustration culminent à nouveau en 1930 avec environ 210 000 exemplaires par semaine. Certains numéros exceptionnels, comme celui de 1929 consacré au Maréchal Foch, sont tirés jusqu'à 650000 exemplaires. Jean-Noël Marchandiau, L'Illustration, 1843-1944 : vie et mort d'un journal, Toulouse, Privat, 1987, p. 325.

72. Pascal Ory, La belle illusion: culture et politique sous le signe du Front populaire, 1935-1938, Paris, CNRS Éditions, 2016.

73. VU coûte 1,5 franc à l'origine, puis 2 francs à partir d'octobre 1930. En 1928, un quotidien coûte 25 centimes. En 1930, les hebdomadaires spécialisés imprimés en héliogravure tels que Match L'Intran sont vendus à 1 franc. Le prix de L'Illustration atteint la même année 4 francs.

74. Serge Berstein, «Les classes moyennes devant l'histoire ", Vingtième Siècle. Revue d'histoire, 37, 1, 1993, pp. 3-12, p. 7.

75. «VU enverra son photographe sur 50 plages pour y prendre des photographies qui paraittront dans $V U$ et sur lesquelles tous nos lecteurs pourront se reconnaître ", VU, 20, $1^{\mathrm{er}}$ août 1928, p. 468. 76. Jean-Claude Daumas, La révolution matérielle: Une histoire de la consommation, Paris, Flammarion, 2018, p. 241.

77. Jean-Claude Daumas, op. cit., p. 260.

78. Marie-Emmanuelle Chessel, op. cit., p. 106.

79. Jean-Claude Daumas, op. cit., p. 241.

80. « Remarques sur un nouveau journal illustré », loc. cit.

81. Ascanio Cecco, «Mobilité et reproductibilité technique au service de la propagande. Les expositions mobiles du plan Marshall ", Transbordeur: photographie histoire société, 2, 2018, pp. 102-113.

82. Les Trains de France, expositions mobiles de grande envergure, Paris, Trains de France, 1928, pp. 8-15.

83. «Au Salon du Foyer ", L'Intransigeant, 24 octobre 1924, p. 2. La section "L'Art au Foyer » présente des appartements modèles conçus par Marcel Temporal, Djo-Bourgeois et André Lurçat. Son comité d'organisation comprend également Francis Jourdain et est présidé par Auguste Perret. Voir $2^{e}$ Salon du Foyer : catalogue officiel, Paris, S. A. M., 1928.

84. Le dispositif du salon de lecture de $V U$ est évoqué dans des coupures de presse mal ou non identifiées conservées par Charles Peignot dans un classeur: Bibliothèque Forney, Archives Peignot, Fonds Charles Peignot, Boîte VIII, 2.

85. Marie-Emmanuelle Chessel, op. cit., p. 67.

86. « Remarques sur un nouveau journal illustré », loc. cit.

87. Sheila Webb, « Art Commentary for the Middlebrow: Promoting Modernism \& Modern Art through Popular Culture-How Life Magazine Brought "The New" into Middle-Class Homes ", American Journalism, 27, 3, 2010, pp. 115-150.

88. Jessica Kelly, «'To Fan the Ardour of the Layman': The Architectural Review, The MARS Group and the Cultivation of Middle Class Audiences for Modernism in Britain, 1933-1940 », Journal of Design History, 29, 4, 2016, pp. 350-365.

89. Reprenant les analyses de Jean-Marie Charon, Jamil Dakhlia souligne la vocation didactique de la presse magazine, qui s'adresse typiquement à un lectorat segmenté en fonction de son style de vie, avec lequel est entretenue une "relation esthétique». Jamil Dakhlia, «Propriétés et 
fonctions de la presse magazine ", dans Claire Blandin (dir.), Manuel d'analyse de la presse magazine, Malakoff, Armand Colin, 2018, pp. 51-65 ; Jean-Marie Charon, « La presse magazine », Réseaux, 1, 105, 2001, pp. 53-78.

90. Françoise Denoyelle, op. cit., p. 117.

91. Annuaire de la presse française et étrangère et du monde politique, Paris, 1935, p. 285.

92. Sylvia Morris, Rage for Fame: The Ascent of Clare Boothe Luce, New York, Random House Trade Paperbacks, 2014, ch. 23 ; Alan Brinkley, The Publisher: Henry Luce and His American Century, New York, Vintage, 2011, ch. VIII.

93. Sophie Kurkdjian, op. cit. (2014), pp. 486-488.

\section{RÉSUMÉS}

L'hebdomadaire VU (1928-1940) est connu pour son iconographie d'inspiration avant-gardiste et son rôle pionnier dans l'histoire du photojournalisme. L'étude ici présentée le resitue au cœur d'un réseau international, constitué, d'une part, d'artistes et de typographes le reliant à l'Union des Artistes Modernes, au Bauhaus et à la Nouvelle Typographie, d'autre part d'entreprises innovantes du secteur des industries médiatiques : la fonderie Deberny \& Peignot, l'agence de publicité Dorland et les publications de Condé Nast. Le design singulier de VU, produit des exigences à la fois esthétiques et marchandes de ses créateur.ice's, dilue des éléments du modernisme artistique dans une modernité visuelle davantage ordinaire, empruntée à la publicité ou à la presse spécialisée. Il semble lui permettre de séduire un segment spécifique du lectorat français de classe moyenne, et de proposer une nouvelle formule médiatique hybride, caractérisée par un contenu éditorial hétéroclite et définie en grande partie par son apparence graphique. Ni revue bourgeoise, ni illustré populaire, $V U$ signale l'avènement $\mathrm{du}$ format magazine, et d'une culture visuelle « moyenne » ou middlebrow.

The weekly newspaper $V U$ (1928-1940) is known for the avant-garde inspirations of its iconography and for its pioneer role in the history of photojournalism. The following study situates it at the heart of an international network, consisting on one hand of artists and typographers linking it to the Union des Artistes Modernes, the Bauhaus and the New Typography, on the other of innovative media companies: the Deberny \& Peignot foundry, the Dorland advertising agency and Condé Nast's publications. VU's unique design, a product of its creators' aesthetic as well as commercial demands, dilutes elements of artistic modernism in a more ordinary visual modernity, borrowed from advertising or the specialised press. It seems to enable it to seduce a specific segment of the french middle-class, and to introduce a new hybrid formula, characterized by its miscellaneous editorial content and, to a great extent, defined by its graphic appearance. Neither a bourgeois journal, nor a popular illustrated weekly, VU signals the rise in France of the magazine format and of a middlebrow visual culture.

\section{INDEX}

Mots-clés : avant-garde, culture de masse, design, middlebrow, modernité 
AUTEUR

LAURA TRUXA

EHESS, CRAL-CEHTA, doctorante laura.truxa@ehess.fr 